\title{
Molecular Dynamics Study of Cl- Permeation through Cystic Fibrosis Transmembrane Conductance Regulator (CFTR)
}

Zhi Wei Zeng ( $\square$ wilson.zeng@mail.utoronto.ca )

The Hospital for Sick Children https://orcid.org/0000-0002-2631-8311

Paul Linsdell

Dalhousie University

\section{Régis Pomès}

Hospital for Sick Children Research Institute: SickKids Research Institute https://orcid.org/0000-00033068-9833

\section{Research Article}

Keywords: CFTR, Cystic fibrosis, Chloride channel, Ion permeation, ABC protein, Channel pore, Hydrophobic gate

Posted Date: August 13th, 2021

DOI: https://doi.org/10.21203/rs.3.rs-771352/v1

License: (c) (i) This work is licensed under a Creative Commons Attribution 4.0 International License. Read Full License 


\title{
Molecular Dynamics Study of $\mathrm{Cl}^{-}$Permeation through Cystic Fibrosis Transmembrane Conductance Regulator (CFTR)
}

\author{
Zhi Wei Zeng ${ }^{1,2}$, Paul Linsdell ${ }^{3}$, Régis Pomès ${ }^{1,2}$ \\ ${ }^{1}$ Molecular Medicine, Hospital for Sick Children, Toronto ON, M5G 0A4, Canada \\ 2 Department of Biochemistry, University of Toronto, Toronto ON, M5S 1A8, Canada \\ ${ }^{3}$ Department of Physiology \& Biophysics, Dalhousie University, PO Box 15000, Halifax NS, B3H 1X5, \\ Canada
}




\begin{abstract}
:
The recent elucidation of atomistic structures of $\mathrm{Cl}^{-}$channel CFTR provides opportunities for understanding the molecular basis of cystic fibrosis. Despite having been activated through phosphorylation and provided with ATP ligands, several nearatomistic cryo-EM structures of CFTR are in a closed state, as inferred from the lack of a continuous passage through a hydrophobic bottleneck region located in the extracellular portion of the pore. Here, we present repeated, microsecond-long molecular dynamics simulations of human CFTR solvated in a lipid bilayer and aqueous $\mathrm{NaCl}$. At equilibrium, $\mathrm{Cl}^{-}$ions enter the channel through a lateral intracellular portal and bind to two distinct cationic sites inside the channel pore but do not traverse the narrow, de-wetted bottleneck. Simulations conducted in the presence of a strong hyperpolarizing electric field led to spontaneous chloride translocation events through the bottleneck region of the channel, suggesting that the protein relaxed to a functionally open state. Conformational changes of small magnitude involving transmembrane helices 1 and 6 preceded ion permeation through diverging exit routes at the extracellular end of the pore. Although permeating $\mathrm{Cl}^{-}$ions remain mostly hydrated, partial dehydration occurs at the binding sites and in the bottleneck. This portion of the pore undergoes wetting prior to $\mathrm{Cl}^{-}$translocation, suggesting that it acts as a hydrophobic gate. The observed $\mathrm{Cl}^{-}$pathway is largely consistent with the loci of mutations that alter channel conductance, anion binding, and ion selectivity, supporting the model of the open state of CFTR obtained in the present study.
\end{abstract}




\section{Keywords:}

CFTR, Cystic fibrosis, Chloride channel, Ion permeation, ABC protein, Channel pore, Hydrophobic gate

\section{Abbreviations:}

MD: molecular dynamics

CF: cystic fibrosis

CFTR: cystic fibrosis transmembrane conductance regulator

ATP: adenosine triphosphate

ABC: ATP-binding cassette

TM: transmembrane (helix if followed by a number)

TMD: transmembrane domain

NBD: nucleotide-binding domain

SASA: solvent-accessible surface area 


\section{Introduction:}

Cystic fibrosis transmembrane conductance regulator (CFTR) is a chloride channel found in cells of epithelial tissues [1-4]. Vital for the secretory function of various tissues, this ion channel regulates the transport of ions and water onto the apical surface of epithelia lining the airways, sweat glands, and the lumen of exocrine organs [5-10]. Loss-of-function mutations in the gene encoding CFTR result in the lethal disease cystic fibrosis (CF). Because many organs rely on secretion for proper function, CF patients experience a wide range of symptoms such as malnutrition and infertility. The lethal consequence of $\mathrm{CF}$ is respiratory failure due to recurring infections, as CF patients' lungs have an impaired ability to secrete mucus needed to rid pathogens [11]. Due to its profound clinical significance, CFTR has attracted much research attention aiming to unravel the mechanism of its function and dysfunction.

CFTR is a member of the ATP-binding cassette $(A B C)$ transporter superfamily, specifically the $A B C C$ exporter subfamily $[1,12]$. Like closely related $A B C$ exporters, CFTR consists of two transmembrane domains (TMDs), which create a transport pathway across the membrane, and two nucleotide-binding domains (NBDs), which bind and hydrolyze ATP to facilitate substrate translocation through the TMDs [13]. CFTR also contains a disordered regulatory domain (R-domain) that can be phosphorylated. Remarkably, CFTR is the only member of the $A B C$ proteins known to function as an ion channel. Whereas a typical $A B C$ transporter utilizes energy from ATP-hydrolysis to actively transport substrates against a transmembrane concentration gradient, CFTR couples its gating cycles to ATP binding and hydrolysis, allowing controlled, rapid movement of ions along the electrochemical gradient [14, 15]. Gating is also regulated by protein kinase $A(P K A)$, which is required to activate the CFTR channel by binding and/or phosphorylating its R-domain [16, 17].

In recent years, near-atomic resolution structures of full-length CFTR were determined by cryo-electron microscopy (cryo-EM) [18-22]. The structures were determined in two distinct functional states: the dephosphorylated, ATP-unbound state (inactive or interburst closed state); and the phosphorylated, ATP-bound state (putative open state). In the inter-burst closed state structure, the cavity of the channel is open to the intracellular 
space but not to the extracellular space, comparable to the "inward-facing" (IF) conformation of closely related $A B C$ transporters $[14,18]$. The dephosphorylated $R-$ domain is found between two TMD-NBD pairs, effectively preventing the two NBDs from dimerizing $[18,19]$. ATP-binding-induced NBD dimerization is required for functional conformational changes to occur in the TMDs [23-25]. Indeed, in the putative open state structure, disengagement of the R-domain, likely due to phosphorylation, allows the NBD dimer to form with bound ATP; the TMDs also adopt a different conformation compared to the closed-state structure [20, 21]. In closely related ABC exporters, ATP binding induces the "outward-facing" (OF) conformation characterized by a wide opening on the extracellular end and closed-off intracellular access [14, 26]. In this IFto-OF, alternating-access transport mechanism, the transporters ensure that the transmembrane cavity is never accessible from both sides of the membrane simultaneously [14, 27]. In contrast, the channel cavity of CFTR is still accessible from the intracellular space when the NBDs are dimerized. Because CFTR functions as an ion channel, its ion conducting state necessarily requires openings on both sides of the membrane. Due to this feature, CFTR has also been described as a "broken transporter" that bears a single gate at the extracellular end of the TMDs $[20,28]$. In electrophysiological records, applying PKA and Mg-ATP to CFTR results in the opening of the channel with a single-channel conductance of 6-10 pS for $\mathrm{Cl}^{-}$ions [19, 29]. However, none of the structures of phosphorylated CFTR in the ATP-bound, NBDdimerized state show an open passage across the TM-region that is sufficiently wide to accommodate water or $\mathrm{Cl}^{-}$ions [20,21]. Therefore, these structures cannot represent the ion-conducting state observed under experimental and physiological conditions. It has been speculated that this NBD-dimerized but closed conformation represents the brief intra-burst closed state observed in electrophysiological records, which may be stabilized by the non-native environment in detergent micelles and requires additional transmembrane helical movements to reach the open state [20]. Molecular dynamics simulations of the phosphorylated, ATP-bound zebrafish CFTR structure revealed that this closed state with dimerized NBDs is stable over hundreds of nanoseconds [30, 31]. Enhanced sampling methods were used to derive putative structures of CFTR with an open pathway permissive of the passage of $\mathrm{Cl}^{-}$ions [31, 32]. However, the molecular 
mechanisms governing the spontaneous opening of the CFTR channel and $\mathrm{Cl}^{-}$ permeation events have yet to be described.

In this study, we present repeated MD simulations of ATP-bound, NBD-dimerized human CFTR in the microsecond time range. In the absence of transmembrane voltage, the extracellular portion of the channel remained largely de-wetted, and ion permeation was not observed. However, spontaneous $\mathrm{Cl}^{-}$translocation events occurred in simulations where a hyperpolarizing transmembrane voltage was applied. The analysis of ion-protein interaction reveals at least two $\mathrm{Cl}^{-}$binding sites in the portion of the channel lumen accessible from the intracellular medium ("inner vestibule") and diverging anion pathways through the narrow extracellular portion of the channel ("bottleneck"). Experimental measurements of ion selectivity are rationalized in light of observed $\mathrm{Cl}^{-}$ interactions with CFTR. Displacements of pore-lining helices occurring under transmembrane voltage are correlated with wetting of the bottleneck region prior to ion translocation, suggesting that the extracellular portion of the channel functions as a hydrophobic gate. 


\section{Results:}

Overview of the channel vestibule

We performed MD simulations of human CFTR in its ATP-bound, NBD-dimerized state (PDB: 6MSM) embedded in a POPC bilayer and solvated in $150 \mathrm{mM} \mathrm{NaCl}$ aqueous solution. In all simulations, water filled the transmembrane channel cavity through a lateral intracellular opening formed by cytosolic extensions of transmembrane (TM) helices 4 and 6 and known as the "cytosolic portal" from structural and functional data (Fig. 1a) $[18,19$, 33]. Chloride ions entered from the intracellular space through the same opening, whereas sodium ions did not enter the cavity (Fig. 1b). This observation suggests that the cytosolic portal imparts selectivity for anions over cations, possibly due to the abundance of positively charged residues at this location [20,34].

Unlike most ion channels, in which the ion permeation pathway tends to be linear and symmetrically shaped, CFTR presents a curved passage to $\mathrm{Cl}^{-}$ions. Two elbow-shaped turns create a short, laterally oriented segment at around $z=-25 \AA$ (Fig. 1b). An apparent barrier opposing $\mathrm{Cl}^{-}$occupancy and passage is found at the extracellular end of the transmembrane domains $(-5 \AA<z<5 \AA$ ), suggesting the presence of an energetic or steric bottleneck (Fig. 1b; Fig. 2d). Lined by TM helices 1, 2, 6, 8, 11, and 12 , this bottleneck region is rich in hydrophobic residues and is the most de-wetted region of the channel pore (Fig. 1c, d; Fig. 2b, c). In the absence of voltage, the ions reached as far as $z=-5 \AA$ into the cavity, but $\mathrm{Cl}^{-}$permeation did not occur. To induce $\mathrm{Cl}^{-}$ permeation, we simulated the same system in the presence of a uniform electric field along the membrane normal. This electric field creates an effective membrane potential of $-500 \mathrm{mV}$ without affecting the structural integrity of the TMDs (Fig. S1). The presence of the electric field increased the average ionic occupancy of the inner vestibule from 1.05 to 1.71 (Fig. 2a, e). Compared to simulations without an electric field, this extra $\mathrm{Cl}^{-}$ ion density occupies the region of the inner vestibule closest to the bottleneck (Fig. 2d, e: $-20 \AA<z<-5 \AA$ ). Despite the high transmembrane voltage, the apparent bottleneck

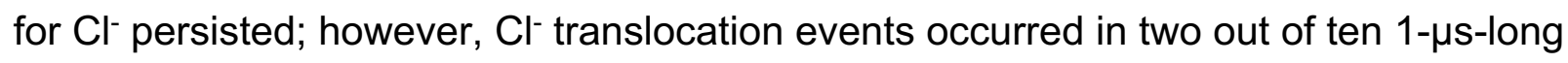
simulations, as discussed in detail below. 


\section{$\underline{\mathrm{Cl}^{-} \text {binding sites in the inner vestibule }}$}

The analysis of the average $\mathrm{Cl}^{-}$distribution in the pore (Fig. 2d) suggests the presence of multiple ion binding sites in the inner vestibule, where protein residues directly contacted $\mathrm{Cl}^{-}$ions through the first solvation shell. In particular, two major binding sites were identified where $\mathrm{Cl}^{-}$ions resided for long durations. The first site is located near the cytosolic portal and consists primarily of residues K190, R248, and R303 (Fig. 3a). The second binding site is located $10-15 \AA$ above the elbow region and consists primarily of K95, R134, and Q98 (Fig. 3a). In the absence of an electric field, site 2 is the farthest location reached by $\mathrm{Cl}^{-}$ions. The presence of an electric field increased the occupancy of site 2 from 0.14 to 0.50 (Fig. 2d, e). Between binding sites 1 and 2, $\mathrm{Cl}^{-}$ bound more transiently to two other sites: one involving R352 and W356, with some contribution from R303; and the other involving primarily R1097 (Fig. S4). Chloride binding resulted in time periods of hundreds of nanoseconds during which the $\mathrm{Cl}^{-}$ion stayed relatively stationary along the channel pathway, even in the presence of voltage (Fig. S7; Fig. S8). Despite that multiple protein residues can bind $\mathrm{Cl}^{-}$ion at either site, on average only one protein residue bound $\mathrm{Cl}^{-}$ions in sites 1 and 2, respectively. Chloride ions at either site remained well hydrated regardless of whether the electric field is present (Fig. 3b).

\section{$\underline{\mathrm{Cl} \text { - permeation through the bottleneck region }}$}

A total of $17 \mathrm{Cl}^{-}$translocation events occurred in runs \#1 and \#2 (Fig. 4; Table S1). These permeation events were closely spaced in time and occurred in three apparent bursts, suggesting interconversions between conductive and non-conductive states. Run \#1 contained one such burst involving $3 \mathrm{Cl}^{-}$permeation events ( $800<t<1000 \mathrm{~ns}$ ) and run \#2 contained two bursts ( $400<t<500 \mathrm{~ns}$ and $600<t<800 \mathrm{~ns}$ ) involving 3 and $11 \mathrm{Cl}^{-}$permeation events, respectively.

The translocation of $\mathrm{Cl}^{-}$through the bottleneck region begins at $z=-5 \AA$. At this location, $\mathrm{Cl}^{-}$ions bind S341 and N1138 from TM6 and TM12, respectively (Fig. 5d). These residues precede the bottleneck region and can be reached by intracellular $\mathrm{Cl}^{-}$ions without leading to translocation, even in the presence of a strong transmembrane voltage (Fig. 4; Fig. S8). Beyond S341, translocating $\mathrm{Cl}^{-}$ions traverse the bottleneck 
region lined by a number of hydrophobic residues including L102, I106, F337, and M1137 (Fig. 5c). Halfway through the bottleneck $(z=0 \AA)$, T338 and T1134 mark the narrowest region of the pore and occasionally coordinate permeating $\mathrm{Cl}^{-}$ions (Fig. 5b). The extracellular end of the bottleneck is lined with several positively charged residues, including R104, R117, R334, and K335, which could facilitate the passage of permeating anions through the bottleneck (Fig. 5a). These cationic residues contribute to the partial dehydration of $\mathrm{Cl}^{-}$by displacing on average about $1.2 \pm 0.6$ water molecules in the first solvation shell of $\mathrm{Cl}^{-}$(Fig. 5e, f). Interestingly, the long sidechain of R334 was even observed to dip into the hydrophobic bottleneck and to bind permeating $\mathrm{Cl}^{-}$ions before they reach T338 (Fig. S6), suggesting that R334 may serve as a chaperone for $\mathrm{Cl}^{-}$translocation.

The $\mathrm{Cl}^{-}$permeation pathway appears to diverge into different routes in the bottleneck region. The point of divergence is located shortly beyond S341. In the first route, $\mathrm{Cl}^{-}$ exited laterally between TM1 and TM6; this route will henceforth be referred to as the "1-6 pathway" (Fig. 6a, d). The 1-6 pathway is bounded by TM helices 1, 6, 8, and 12. In an alternative route, $\mathrm{Cl}^{-}$exited between TM helices 1 and 12, which defines the "1-12 pathway" bounded by TM helices 1, 2, 6, 8, 11, and 12 (Fig. 6c, f). The third permeation route, which is intermediate between the other two, is lined by the same six TM helices in approximate hexagonal arrangement (Fig. 6b, e). The three different permeation routes also differ in which sets of extracellular residues coordinate translocating $\mathrm{Cl}^{-}$ions: R334, K335, and R104 in the 1-6 pathway (Fig. 5a); R334, Y914, and Y917 in the intermediate pathway (Fig. 7c); R117 and polar groups of extracellular loops in the 1-12 pathway (Fig. 7b).

\section{Structural features of the open pore}

In the simulations in which $\mathrm{Cl}^{-}$permeation events occurred, changes in the distances separating pore lining helices in the bottleneck region were correlated with transitions from the non-conductive state to conductive states (Fig. 8). In run \#1, ion permeation may be facilitated by TM6 moving away from other pore lining helices (Fig. 8d). In particular, the distance $d_{1-6}$ increased from $\sim 9 \AA$ to $\sim 12 \AA$ before the permeation burst 
starting at $800 \mathrm{~ns}$ (Fig. 9a), creating a pore large enough for ion exit through the 1-6 pathway (Table S1).

In run \#2, ion permeation was likely facilitated by TM1 moving away from other pore lining helices (Fig. 8e). The distances $d_{1-8}$ and $d_{1-12}$ increased at $\sim 400$ ns (Fig. 9e, h) even as $d_{1-6}$ decreased before reverting to larger values during the second ion burst period (Fig. 9b). The decreased TM1-TM6 separation may explain that all three permeant $\mathrm{Cl}^{-}$ions took the 1-12 pathway during the first ion burst (Table S1), during which $d_{1-12}$ peaked (Fig. S3). The second ion burst featured a large $d_{1-12}$ and a further increase in $d_{1-8}$ (Fig. 9e, h). During this period, permeant $\mathrm{Cl}^{-}$ions followed mostly the intermediate and 1-6 pathways (Table S1). After the second ion burst, $d_{1-6}$ increased further while $d_{1-12}$ decreased (Fig. 9b, h; Fig. S3), suggesting that the channel may be undergoing a transition towards a state in which the 1-6 pathway is the exclusive route, similar to that observed in run \#1.

\section{Hydration of the bottleneck region}

The observation of partially hydrated $\mathrm{Cl}^{-}$ions permeating through the CFTR channel suggests that a hydrated bottleneck region is a prerequisite for ion conduction. Overall, the average number of water molecules in the bottleneck increased slightly when the electric field was present (Fig. 10a), a phenomenon seen in hydrophobic gates and known as "electric field-induced wetting" [35]. The overall degree of hydration in run \#1 was greater than that of all other simulations conducted with an electric field. All ion permeation events in this simulation run occurred when hydration was relatively high after $d_{1-12}$ increased to permit water permeation through the 1-12 pathway (Fig. 4; Fig. 9g; Fig. 10b). Likewise, the time dependence of hydration in run \#2 was strongly correlated with ion permeation events. The first and second permeation bursts occurred following major increases in hydration at $t \sim 400 \mathrm{~ns}$ and $t \sim 600 \mathrm{~ns}$, respectively (Fig. 4;

Fig. 10c). Permeation occurred during periods of wetting of the bottleneck region, which provided a hydrated pathway for ion translocation.

The coincidence of pore wetting and ion permeation events is reminiscent of hydrophobic gates in a large variety of other ion channels [36, 37]. To locate a putative hydrophobic gate in CFTR, we analyzed hydrophobicity along the permeation pathways. 
A short but highly hydrophobic "gasket" consisting of residues L102, I106, and F337 constitutes the most hydrophobic and narrowest region within the 1-6 pathway (Fig. $11 a, b)$. The hydrophobicity of this region decreases drastically when sustained hydration and permeation occur due at least in part to the penetration of the side chain of R334 (Fig. S6). Similarly, residues L102, I105, M1137 constitute a highly hydrophobic region within the intermediate pathway (Fig. 11e-f). This pathway is too narrow to be detected until it becomes available with a pore diameter of at least $4 \AA$ throughout (Fig. 11f). In contrast, the 1-12 pathway does not have a distinct, sharply hydrophobic region; instead, the entire pathway is somewhat hydrophobic (Fig. 11c, d). During permeation, the entire pathway appears widened, with the narrowest region widening by about $1-2 \AA$ in diameter.

Drastic changes in hydration around the gasket region occurred during transitions between non-conducting and conducting states (Fig. 10d-i). In run \#1, P99 and L102 became substantially more and less hydrated, respectively, prior to the ion permeation burst (Fig. 10d, e). Since L102 was much more water accessible when water permeation occurred through the 1-12 pathway (Fig. 10e; $500<t<800 \mathrm{~ns}$ ), the decrease in water accessibility of L102 might coincide with the closure of the 1-12 pathway and the opening of the 1-6 pathway. These observations suggest that differential extent of wetting of these residues is correlated with the availability of different permeation pathways. Accordingly, L102 is more water exposed than P99 when $\mathrm{Cl}^{-}$permeation occurs through the 1-12 and intermediate pathways in run \#2 (Fig. $10 \mathrm{~g}$, h). Furthermore, 1106 is more hydrated in run \#2 than in run \#1, especially during the second ion permeation burst (Fig. 10i), which may be a hallmark of a wide-open gate supporting all permeation routes. In summary, wetting of hydrophobic residues within the bottleneck region precedes permeation events regardless of permeation routes; however, which residues undergo wetting depends on the permeation pathway being activated. 


\section{Discussion:}

\section{Comparison with previous structural modelling studies}

The elucidation of both open and closed state structures of the CFTR ion channel, as well as the transitions between the different states, are crucial for the understanding of CFTR function and dysfunction. Despite major progress made in structure determination, near-atomic resolution cryo-EM structures of this protein could not explain how it allows permeation of $\mathrm{Cl}^{-}$ions [18-22]. In the present work, we performed atomistic MD simulation of human CFTR in a lipid bilayer. In the presence of a hyperpolarizing transmembrane voltage, spontaneous $\mathrm{Cl}^{-}$translocation events occurred through divergent pathways in the extracellular portion of the pore.

Prior to this work, numerous in silico studies involving molecular simulations of homology models of CFTR were devoted to providing a picture of the channel in its open conducting state, both before and after the advent of high-resolution experimental structures (reviewed in [32]. A major limitation of these homology models is the use of homologous template $A B C$ transporters sharing low sequence identity with CFTR [32, 38-40]. In addition, an unexpected feature revealed by the near-atomic resolution cryoEM structures is that TM8 is partially unwound in the middle of the membrane and split into two helical segments [18]. As a result, the extracellular segment of TM8 participates in lining the pore, in stark contrast to typical, symmetric $A B C$ transporters in which TM7 lines the translocation pathway. Recently, using substituted cysteine accessibility mutagenesis (SCAM) and cysteine crosslinking, Negoda and coworkers found that TM8 contains residues lining the narrow region of the pore in close proximity to TM1 and TM6 in an open CFTR channel [41]. This feature of TM8 is consistent with the nearatomic resolution experimental structures but is not captured by existing homology models. More recently, Farkas and coworkers performed equilibrium MD simulations on the cryo-EM structure of ATP-bound, NBD-dimerized zebrafish CFTR, which revealed a transient open pathway through the channel allowing water permeation [31]. Metadynamics was used to direct $\mathrm{Cl}^{-}$through the bottleneck region, revealing two putative energetically favourable pathways [31]. Our simulation results on human CFTR, also performed on the ATP-bound, NBD-dimerized state, support the prediction of 
multiple $\mathrm{Cl}^{-}$exit pathways and reveal how conformational rearrangements of pore-lining helices give rise to different routes of permeation.

The peculiarity of the TM8 structure attracted attention due to its potential role in the gating of CFTR $[18,30]$. It was speculated that movements of TM8 are key to transitions from a non-conducting state to a conducting state in the presence of ATP and dimerized NBDs [20]. In contrast, Fay and coworkers obtained a lower-resolution cryo-EM structure of engineered chicken CFTR in an ATP-bound form [42]. This structure, which was determined to have a high channel opening probability, does not have a "broken" TM8. In the current study, the conformation of TM8 as it appears in the near-atomic resolution cryo-EM structures is stable over microseconds of simulation time, with the RMSD of its extracellular segment from the PDB structure reaching up to $\sim 2 \AA$ after 1 $\mu$ s (Fig. S1). This observation is consistent with the findings from a previous MD simulation study of zebrafish CFTR supporting the conformational stability of TM8 [30]. Accordingly, spontaneous $\mathrm{Cl}^{-}$permeation events occurred in the presence of an electric field without major conformational changes in TM8, such as the rotation of its extracellular segment or the restoration of its helical defect.

\section{Chloride binding sites}

The location and physico-chemical nature of $\mathrm{Cl}^{-}$binding sites in CFTR have been somewhat contentious $[43,44]$. Experimental studies conducted with negatively charged channel blockers point to the presence of anion binding sites inside the cavity $[45,46]$. From our simulations, we identified two major binding sites that are consistent with the experimental findings: site 1, near the cytoplasmic portal, consisting of residues K190, R248, and R303; and site 2, deep inside the inner vestibule of the pore, consisting of K95, Q98 and R134 (Fig. 3a). Several of the residues that contribute to these two binding sites have previously been shown to interact with channel-blocking anions, which have been suggested either to occupy a superficial site close to the cytoplasmic portal $[33,46]$ or to enter deep into the cavity and interact with the positive charge of K95 [45]. Furthermore, the reduced conductance associated with mutations that remove positive charges in these binding sites suggests that $\mathrm{Cl}^{-}$binding to these sites is an important feature of its permeation mechanism [34, 47, 48]. These two inner 
binding sites are located in regions where $\mathrm{Cl}^{-}$occupancy is likely to be importantnamely, at the cytoplasmic entrance of the pore (site 1) and near the entrance of the bottleneck (site 2). Indeed, $\mathrm{Cl}^{-}$ions are most likely to occupy site 1 regardless of electric field, while the presence of an electric field increases the affinity of the middle of the pore for anions, as seen by the increase in $\mathrm{Cl}^{-}$occupancy at site 2 (Fig. 2d, e).

Even though simultaneous binding to multiple sidechains of site 2 does occur occasionally, usually only one residue binds $\mathrm{Cl}^{-}$at a time, suggesting that $\mathrm{Cl}^{-}$-binding residues are loosely organized at the binding site. Accordingly, it was found by mutagenesis that the positive charge of $\mathrm{K} 95$ can be moved to a nearby location, such as I344, V345, or M348, without abrogating channel function [49, 50]. Similarly, the positive charges on K190 and R303 can be moved to nearby locations, such as N186 and L197, with minimal reduction in $\mathrm{Cl}^{-}$conductance [51].

\section{Ion permeation through the bottleneck region}

Consistent with functional and SCAM studies, in the ion-conducting conformations of CFTR, the ionic pathway through the bottleneck is lined primarily by TM helices 1, 6, 8, and 12 (Fig. 1C) [41, 43, 52]. TM helices 2 and 11 also contribute to lining the permeation pathway, albeit to a lesser extent (Fig. 1c; Fig. 6). While there has been some evidence suggesting that TM11 lines the narrow region of the permeation pathway, evidence supporting the same role for TM2 is lacking [43]. Our simulations show two key residues by which TM2 could functionally contribute to the $\mathrm{Cl}^{-}$permeation pathway: R134, which contributes to binding site 2; and R117, which binds $\mathrm{Cl}^{-}$ions permeating through the 1-12 pathway. However, neither of these residues is located within the narrow bottleneck region. Furthermore, neither TM2 nor TM11 appear to make any contribution to the 1-6 pathway.

The simulations show that $\mathrm{Cl}^{-}$entering the bottleneck region from the intracellular side first contact S341 (Fig. 5d, f). The hydroxyl group of S341 participates in $\mathrm{Cl}^{-}$solvation, which might be important given that the S341A mutant is associated with a large decrease in $\mathrm{Cl}^{-}$conductance [53]. Moreover, mutation S341K also causes greatly reduced conductance, highlighting the importance of this side chain to the permeation process [50]. Beyond S341, the narrow permeation pathway is lined by hydrophobic 
sidechains including L102, I106, F337, and M1137 (Fig. 5c). Functional and SCAM data suggest that L102 and F337 contribute to the narrowest part of the open pore [54, 55]. Mutations of T338 show strongly size-dependent effects on conductance, consistent with the observation that this residue is located in the narrowest part of the bottleneck $[56,57]$. At the extracellular end of the bottleneck, $\mathrm{Cl}^{-}$ions interact with R104, R117, R334, and K335 (Fig. 5a; Fig. 7b, c). The positive charges at R104, R334, and K335 have each been shown to interact with extracellular anions, and charge-neutralizing mutations at these residues reduce $\mathrm{Cl}^{-}$conductance [52]. In our simulations, R334 was seen to interact with $\mathrm{Cl}^{-}$even before it reached the extracellular space. Finally, $\mathrm{Cl}^{-}$ions exiting via the intermediate pathway interact with Y914 and Y917, mutations of which were shown to affect both $\mathrm{Cl}^{-}$permeation and channel gating [55].

Conformational fluctuations leading to the open conformation of the channel involve relative movements of TM1 and TM6 (Fig. 8). Cysteine cross-linking studies suggested that the extracellular ends of TM1 and TM6 separate from each other when the channel opens [58], which is consistent with the 1-6 permeation pathway observed in this study. TM1 movements best explain gate wetting and ion translocation. These movements may be facilitated by a helical defect at residue P99. Consistent with this hypothesis, mutations that favour helicity, such as P99A and P99L, are both loss-of-function mutations [59]. TM6 movements may also facilitate ion translocation, as seen in run \#1, albeit to a lesser degree. In contrast, we did not observe noticeable movements of TM8 and TM12 as proposed by Zhang et al. [20].

\section{$\underline{\text { Putative explanations of lyotropic permeability selectivity }}$}

Like most anion channels, CFTR shows lyotropic permeability selectivity, meaning that lyotropic anions with a relatively low free energy of hydration have higher permeability than kosmotropic anions, which retain water in their hydration shell more strongly [60, 61]. One implication of lyotropic permeability selectivity is that partial dehydration is an important aspect of the anion permeation process. Consistent with this hypothesis, $\mathrm{Cl}^{-}$ ions lose water molecules from their first solvation shells as they pass through the bottleneck region (Fig. 5e). This finding suggests that the bottleneck might form the lyotropic "selectivity filter," where the relative permeability of different anions is 
predominantly determined. Amongst all the pore-lining residues studied, only mutagenesis of F337 and, to a lesser extent, L102, have been shown to disrupt the normal lyotropic selectivity pattern [55, 61]. These non-polar residues form a narrow, hydrophobic region of the open pore where $\mathrm{Cl}^{-}$ions are partially dehydrated (Fig. 5e-f). It is conceivable that partial dehydration might favour the entry (and therefore permeation) of more lyotropic anions, thus explaining the lyotropic permeability selectivity pattern.

\section{Diverging ion translocation pathways in the bottleneck region}

Our simulations reveal multiple $\mathrm{Cl}^{-}$exit pathways to the extracellular space (Fig. 6). In support of both 1-6 and 1-12 pathways, charge-neutralizing mutations at R104, R334, $\mathrm{K} 335$, and R117 lead to reduced channel conductance [62, 63]. In addition to these two permeation pathways, a less distinguishable, intermediate pathway (Fig. 6b, e; Fig. 11e) involving direct $\mathrm{Cl}^{-}$coordination by tyrosine sidechains $\mathrm{Y} 914$ and $\mathrm{Y} 917$ was observed when the gate was most widely open (Fig. 7c). In support of the intermediate pathway, a number of mutations of these tyrosine residues lead to reduced channel conductance [41].

Given the small number of ion permeation events in the simulations, we cannot assess the relative stability and functional importance of each pathway. The existence of these different pathways may not correspond to observable changes in the functional properties unless specific blockage of individual putative pathways can be achieved. Mutagenesis of some residues in the outer mouth of the pore, including R117, had relatively minor effects on conductance, possibly because these residues contribute to the pathway taken by only a subset of all permeating $\mathrm{Cl}^{-}$ions $[63,64]$. Furthermore, rapid fluctuations in the conformation and relative arrangement of pore-lining helices governed the transitions between the different pathways over a 100-ns timescale (Table 1: simulation run \#2: $600<t<800 \mathrm{~ns}$ ). One implication of TM-helix movements is that the extracellular part of this pore is structurally dynamic, which might explain why we did not identify a clearly defined "outer vestibule" region as suggested in the classical description of the architecture of CFTR pore [52]. 


\section{$\underline{\text { A hydrophobic gate with unusual features }}$}

We have uncovered evidence suggesting that the extracellular bottleneck region of the CFTR pore functions as a hydrophobic gate. Hydration of the hydrophobic bottleneck is greater in the presence of an electric field (Fig. 10a, j-I). Electric-field-induced permeation of water and ions was observed in simulations of hydrophobic nanopores [65]; more recently, wetting of hydrophobic gates was demonstrated in simulations of biological ion channels in the presence of a strong electric field $[35,66,67]$. It has been suggested that the presence of an electric field can alter the liquid-vapour equilibrium of water inside hydrophobic gate, resulting in an increased probability of wetting [66]. This effect might have contributed to water and ion permeation in our simulations. However, the wetting transition observed in the simulations can also be explained in terms of structural changes. Increasing both the polarity and the diameter of the gate are common features of hydrophobic gating [68]. In our simulations, both of these events occurred due to movements of helices and changes in sidechain conformations, both of which may be induced by the electric field.

Despite similarities to other hydrophobic gates, the proposed hydrophobic gate of CFTR also has some peculiarities. First, it is less distinctively shaped due to the presence of multiple permeation pathways. While the 1-6 pathway resembles a typical ion channel pore with a short hydrophobic segment, the 1-12 pathway does not seem to have a distinct gating region, yet it is overall lacking in polar residues and, as expected, it undergoes dilation prior to ion permeation. Second, partial dehydration of the permeant ion occurs as it passes through the hydrophobic bottleneck. The fact that sidechains such as R334 can participate in the solvation of permeant ions complicates its categorization as a hydrophobic gate. Curiously, size-reduction mutation F337A and hydrophobicity-reducing mutation L102T both result in reduced channel conductance $[47,55]$. Along with many other intriguing effects of mutations in residues of the bottleneck region $[44,52]$, these are some of the transport properties of $\mathrm{Cl}^{-}$in $\mathrm{CFTR}$ that remain to be unravelled. 


\section{Conclusions}

Using MD simulations, we have characterized $\mathrm{Cl}^{-}$permeation events through the human CFTR channel in the presence of transmembrane voltage. Traversing $\mathrm{Cl}^{-}$ions interacted with pore lining residues that are critical for the ion conductance, supporting this model of open CFTR channel. The spontaneous opening of the channel from the cryo-EMdetermined state involves conformational rearrangements of TM helices 1 and 6 , which line the extracellular hydrophobic bottleneck region of the pore. The wetting/de-wetting properties of the bottleneck region evoke hydrophobic gating, adding CFTR to the list of ion channels that utilize this physico-chemical mechanism to modulate ion permeation.

Despite the high applied voltage, the small number and relatively short duration of conductive bursts observed in the present study resulted in a small number of ion permeation events. As such, our results do not permit a reliable estimate of the conductance of the open channel. In order to estimate $\mathrm{Cl}^{-}$conductance and compare it to single-channel conductance measurements, longer simulations of the open state will be required to compute steady-state current-voltage relationships at lower, physiological voltages. These future studies will aim to clarify the relative stability of the diverging permeation pathways observed in the present study and provide a meaningful validation of our structural model of the conducting state of CFTR. 


\section{Methods:}

\section{Molecular System}

The structural model of phosphorylated, ATP-bound human CFTR (PDB: 6MSM) was used for all simulations [21]. Residues that were missing in the PDB structure include the loops connecting each TMD-NBD pair (410-434, 1174-1201), the R-region (638844), the extracellular loop between TM7 and TM8 (890-899; also known as the extracellular loop 4), and the segment at the C-terminal end of NBD2 (1452-1489). These missing segments were not modelled in this study, resulting in our structural model consisting of five peptide chains. All chains were acetylated at the $\mathrm{N}$-termini and amidated at the C-termini into primary amides. Mg-ATP moieties from the original PDB structure were found at the NBD interface and were retained for the simulations. All other species present in the PDB structure were removed if they were not known regions of CFTR or Mg-ATP. These species include cholesterol, phospholipids, and the helix at the TMD-NBD interface believed to be part of the R-domain.

Before embedding the CFTR protein into lipids, the PPM server of Orientations of Proteins in Membranes (OPM) database was used to determine the starting position of the lipid bilayer [69]. The model for CFTR with Mg-ATP bound was then embedded in a POPC bilayer and solvated in water with $150 \mathrm{mM} \mathrm{NaCl}$ using the CHARMM-GUI server $[70,71]$, with no water or ions allowed inside the channel pore. The hexagonal periodic unit cell configuration was chosen with starting dimensions: $a=b=11 \mathrm{~nm}, c=18.5 \mathrm{~nm}$, $\alpha=\beta=90^{\circ}$, and $\gamma=120^{\circ}$. This procedure resulted in a total of 255 POPC molecules added around the protein within a periodic image.

\section{Simulation setup and protocol}

All MD simulations were conducted using GROMACS 2018 or 2019 [72]. The CHARMM36 forcefield was used for protein, lipids, ions, ATP, together with the TIP3P water model [73-76]. Simulations were run in the $N p T$ ensemble $(T=300 \mathrm{~K}, p=1 \mathrm{~atm})$ at $2 \mathrm{fs}$ integration timesteps. Constant temperature was maintained using the NoséHoover thermostat $\left(\tau_{\top}=0.5\right)[77,78]$; constant pressure was maintained using the Parrinello-Rahman barostat $\left(\tau_{p}=2.0\right)[79,80]$. Semi-isotropic pressure coupling was 
used, with isothermal compressibility set to $4.5 \times 10^{-5}$ bar $^{-1}$ both in the $x y$-plane and along the z-axis. Nonbonded interactions were calculated using Verlet neighbor lists [81, 82]. Lennard-Jones interactions were cut off at $1.2 \mathrm{~nm}$ and a force-based switching function with a range of $1.0 \mathrm{~nm}$ was used. The particle-mesh Ewald (PME) method was used to compute electrostatic interactions with a real-space cut-off of $1.2 \mathrm{~nm}[83,84]$. The LINCS algorithm was used to constrain covalent bonds involving $\mathrm{H}$ atoms [85].

The simulation system was first subjected to steepest descent energy minimization until maximum force dropped below $1000 \mathrm{~kJ} / \mathrm{mol} / \mathrm{nm}$. Random velocities were generated at the beginning of the $N p T$ equilibration phase, which was conducted in three 10-ns stages, successively with protein heavy atoms, protein backbone atoms, and protein Ca atoms restrained (with force constants of $1000 \mathrm{~kJ} / \mathrm{mol} / \mathrm{nm}^{2}$ in $x, y$, and $z$ directions). After the $N p T$-equilibration phase, the simulation was divided into two groups for the production run. In one group, the simulation was conducted in NpT ensemble at the same temperature and pressure without applied restraints. In the other group, a uniform electric field of magnitude $32 \mathrm{mV} / \mathrm{nm}$ was added along the $z$-axis, normal to the lipid bilayer and pointing towards the intracellular side (negative along $z$-axis). During the production runs, the $z$-dimension of the simulation box (i.e. parameter $c$ of unit cell) equilibrated to around $16.5 \mathrm{~nm}$, which gave rise to a transmembrane voltage of approximately $-500 \mathrm{mV}$. This approach has been used to generate a membrane potential in MD simulations without having to maintain asymmetric ion concentration on both sides of the membrane [86, 87]. In both sets of simulations, new random velocities were generated at the beginning of production runs. Ten 1- $\mu$ s-long simulation runs were produced from each set of conditions.

\section{Analysis of simulation data}

All molecular snapshots of CFTR from production run trajectories were aligned to the first frame (i.e. the configuration at the end of the equilibration phase) through minimization of RMSD of Ca atoms of TMDs.

All the visualizations and renderings of molecular representations of proteogenic components and ions were done with VMD [88]. The origin of the $z$-axis was chosen to be the position of the $\mathrm{C} \alpha$ atom of T338. To identify ions located inside the channel pore, 
a simple cylindrical method was used to define the boundaries. The $\mathrm{C} \alpha$ atoms of porelining helices (TM1, 2, 6, 8, 11, and 12) located within $-40<z<20 \AA$ were selected. The centre-of-mass of these $\mathrm{C} \alpha$ atoms in the $x y$-plane was used as the center of the cylinder. The average distance in the $x y$-plane from the center to these $\mathrm{C} \alpha$ atoms was used as the radius.

Analyses of the ions inside the channel (axial position and solvation shell), the structure of pore-lining helices, the hydration of the bottleneck region (including solventaccessible surface area) were done with homemade Tcl scripts aided by VMD and/or python scripts aided by MDTraj [88, 89]. In particular, to analyze ion binding and solvation, a molecule or residue is considered to form a direct contact with a $\mathrm{Cl}^{-}$ion if it contains any atom within $3 \AA$ of the ion. This cut-off criterion is based on radial pair distribution functions computed using VMD (Fig. S2). According to the distribution functions, the closest contacting atoms are most likely hydrogen atom. The hydropathy and channel path radius analysis were aided by MOLE2 [90]. 


\section{Figure Captions:}

Fig. 1 Overview of the CFTR channel pore. (A) Simulation snapshot showing the waterfilled channel cavity (inner vestibule). The inner vestibule is accessible to water from the intracellular space through the "cytosolic portal" (cyan arrow) formed by TM helices 4 and 6. Phosphorus atoms (cyan spheres) of POPC headgroups indicate membrane position. Bulk water and lipid tails are omitted for clarity. (B) Spatial view of occupancy of $\mathrm{Cl}^{-}$ions (green spheres) inside the channel cavity from many timesteps overlaid. (C) Cartoon representation of TM helix segments lining the bottleneck region of the pore together with $\mathrm{Cl}^{-}$ions drawn from multiple timesteps. (D) The bottleneck region of the pore is lined by many hydrophobic residues (grey), disfavouring the presence of water (red surfaces). Selected hydrophilic/charged residues (cyan) are also shown.

Fig. 2 Effect of transmembrane voltage on water and $\mathrm{Cl}^{-}$occupancy of the CFTR channel. (A) Probability distribution of the number of $\mathrm{Cl}^{-}$ions occupying the inner vestibule (blue) without and (green) with applied voltage. Error bars represent bootstrapped $99 \%$ confidence intervals. (B) Snapshot of the CFTR channel filled with water molecules used as positional reference for (C)-(E). (C) Average number density of water molecules ( $N_{\text {water }}$ ) in the channel vestibule computed along the z-axis. The bottleneck region is highlighted in yellow shading. (D) Average population density of $\mathrm{Cl}^{-}$ ions, $\rho\left(\mathrm{Cl}^{-}\right)$, inside the channel pore (blue) without and (green) with $-500 \mathrm{mV}$ transmembrane voltage. The $\mathrm{Cl}^{-}$occupancy of the region $-20<z<0 \AA$ is enhanced by voltage. (E) Cumulative population densities of $\mathrm{Cl}^{-}$ions, $n\left(\mathrm{Cl}^{-}\right)$, inside the channel pore computed by integrating $\rho\left(\mathrm{Cl}^{-}\right)$from (D).

Fig. 3 Chloride solvation in the inner vestibule. (A) Left: overview of the $\mathrm{Cl}^{-}$permeation pathway with overlaid ions (green spheres) from simulations in the presence of voltage with TM helices 1 (blue), 2 (red), 3 (cyan), 4 (orange), 6 (tan), 8 (lime), 11 (purple), and 12 (yellow). The locations of two $\mathrm{Cl}^{-}$binding sites 1 and 2 are indicated in dashed boxes. Right: close-up views of binding sites 1 and 2 . Amino acid sidechains in direct 
contact with $\mathrm{Cl}^{-}$ions (green spheres) are shown. The labels of positively charged residues, including K246 and K370 from the cytosolic portal, are shaded in indigo. (B) Coordination of $\mathrm{Cl}^{-}$ions inside the channel cavity from simulations with voltage. The average numbers of protein residues (black) and water molecules (red) in the first solvation shell of $\mathrm{Cl}^{-}$ions $\left(n_{\text {solv }}\right)$ along the intracellular pathway are shown together with their sum (green). Shading represents $99 \%$ bootstrapped confidence intervals. Error bars are largest in the bottleneck region, which is least visited.

Fig. 4 Spontaneous permeation of $\mathrm{Cl}^{-}$ions in the CFTR channel under applied voltage. Left: overview of the $\mathrm{Cl}^{-}$permeation pathway with overlaid ions (green spheres) from simulations in presence of voltage highlighting TM helices 4 (orange) and 6 (tan). Right: time series of axial $\mathrm{Cl}^{-}$ion positions from the two simulation runs in which permeation occurred $(-500 \mathrm{mV})$. Ion permeation events are indicated by traces passing through the bottleneck region. Individual $\mathrm{Cl}^{-}$ions are distinguished by color for clarity.

Fig. 5 Chloride translocation pathway through the bottleneck region. Top center: overview of the $\mathrm{Cl}^{-}$permeation pathway with overlaid ions (green spheres) from simulations in the presence of voltage with TM helices 1 (blue), 2 (red), 3 (cyan), 4 (orange), 6 (tan), 8 (lime), 11 (purple), and 12 (yellow). (A)-(D) Close-up views showing the residues interacting with permeating $\mathrm{Cl}^{-}$ions. The labels of positively charged residues are shaded in indigo. (E) Coordination of $\mathrm{Cl}^{-}$ions inside the channel cavity from simulations with voltage. The average numbers of protein residues (black) and water molecules (red) in the first solvation shell of $\mathrm{Cl}^{-}$ions $\left(n_{\text {solv }}\right)$ along the intracellular pathway are shown together with their sum (green). Shading represents $99 \%$ bootstrapped confidence intervals. Chloride ions are less hydrated in the bottleneck region (yellow shading). (F) Significant amino-acid contributions (various colours) to the protein solvation number $n_{\text {solv }}$ (black). 
Fig. 6 The three different pathways of $\mathrm{Cl}^{-}$permeation through the extracellular bottleneck. (Top row) Side views and (bottom row) top views from extracellular space. Pore-lining helices are colour-coded as indicated. Chloride ions (cyan) overlaid from timesteps during translocation. (A), (D) 1-6 pathway; (B), (E) intermediate pathway; (C), (F) 1-12 pathway.

Fig. 7 Outer $\mathrm{Cl}^{-}$binding sites at the extracellular mouth. Boxed regions in the overview of the pore (A) are expanded in insets showing $\mathrm{Cl}^{-}$exit binding sites for (B) the 1-12 pathway and (C) the intermediate pathway. TM helices 1 (blue), 2 (red), 3 (cyan), 4 (orange), 6 (tan), 8 (lime), 11 (purple), and 12 (yellow) are highlighted. Residue sidechains interacting with $\mathrm{Cl}^{-}$ions are shown.

Fig. 8 Structural fluctuations of TM helices lining the extracellular bottleneck region. (A) Top view of the pore from the extracellular side highlighting amino acid residues of the pore-lining helices used to track TM helix movements. (B)-(C) Distributions of $x y$ positions of the extracellular ends of TM helices 1, 6, 8, and 12 in the absence and presence of transmembrane voltage. (D)-(E) Time evolution of TM helices 1, 6, 8, and 12 in conductive simulation runs \#1 and $2(-500 \mathrm{mV})$. In run \#1, a brief departure of TM6 away from the other helices is seen towards $1000 \mathrm{~ns}$. In run \#2, TM1 shows significant fluctuations of up to $\sim 5 \AA$ from its average location.

Fig. 9 Time evolution of interhelical distances between TM 1 and other pore-lining helices at the extracellular end. Interhelical distances are computed as the distances between $\mathrm{C} \alpha$ atom pairs of the following residues: $d_{1-6}$ : I106-R334 (top row); $d_{1-8}$ : I106Y914 (middle row); and $d_{1-12}$ : I106-I1131 (bottom row). (A), (D), (G) Time series of interhelical distances for run \#1 (orange traces); (B), (E), (H) same for \#2 (pink traces). Chloride translocation events (see Fig. 4) are indicated as vertical lines. (C), (F), (I) Distributions of interhelical distances in the absence (blue) and in the presence (green) of an electric field. 
Fig. 10 Analysis of hydration of the extracellular bottleneck region. (A) Distributions of the number of water molecules in the bottleneck region, $N_{\text {water }}$ (bottleneck), from nonconductive simulations in the presence (green) and absence (blue) of TM voltage. Results computed from conductive simulation runs \#1 and \#2 are shown separately. (B)-(C) Time evolution of $N_{\text {water }}$ (bottleneck) for simulation runs \#1 (orange) and 2 (pink). (D)-(I) Time series of solvent accessible surface areas (SASA) of residues P99, L102, and I106 found in the narrowest region of the bottleneck for simulation runs \#1 [(D)-(F)] and $2[(\mathbf{G})-(\mathbf{I})]$. The time series suggest temporal correlation with ion permeation. (J)-(L) Distributions of SASA of these residues in the absence (blue) and in the presence (green) of TM voltage are shown on the rightmost column. lon translocation events (see Fig. 4) are indicated as vertical lines.

Fig. 11 Structural and physico-chemical analysis of diverging permeation pathways through the extracellular bottleneck region. (A) 1-6, (C) 1-12, and (E) intermediate pathways are visualized as translucent closed surfaces. Estimates of width and hydrophobicity along the 1-6 (B), 1-12 (D), and intermediate pathways (F); $R$, local radius and $\zeta$, curvilinear distance along the pathway. The comparison of closed and open states shows reduced hydrophobicity and/or increased width of the permeation pathways. Note that the intermediate pathway in the "closed" state could not be detected and analyzed. The approximate ranges of pathway-lining residues are indicated for each of the three pathways. 


\section{Acknowledgements:}

We thank Christine Bear for providing feedback on early results. This work was supported by Canadian Institutes of Health Research grant MOP130461. MD simulations and analyses were enabled by supercomputing resources and support provided by SciNet (www.scinet.ca) and Compute Canada (www.computecanada.ca).

\section{Declarations:}

Funding: This work is supported by Canadian Institutes of Health Research grant MOP130461 (R.P.) and SickKids Restracomp (Z.W.Z.).

Conflicts of Interest: The authors declare no conflict of interest.

Availability of data and material: The MD simulation datasets are available from the corresponding author(s) upon reasonable request.

Code availability: Selected analysis scripts, parameter files used to reproduce the MD simulations, and parameter files used in MOLE2 analysis will be provided through Github upon publication or request during review.

Authors' contributions: Z.W.Z., R.P. designed and performed the MD simulation study; Z.W.Z. prepared the figures and performed analyses with R.P. and P.L; all authors contributed to interpreting the results and writing the manuscript. 


\section{References:}

1. Riordan, Rommens J, Kerem B, et al (1989) Identification of the cystic fibrosis gene: cloning and characterization of complementary DNA. Science 245:10661073. https://doi.org/10.1126/science.2475911

2. Berger HA, Anderson MP, Gregory RJ, et al (1991) Identification and regulation of the cystic fibrosis transmembrane conductance regulator-generated chloride channel. Journal of Clinical Investigation 88:1422-1431.

https://doi.org/10.1172/JCl115450

3. Welsh MJ, Smith AE (1993) Molecular mechanisms of CFTR chloride channel dysfunction in cystic fibrosis. Cell 73:1251-1254. https://doi.org/10.1016/00928674(93)90353-R

4. Anderson M, Gregory R, Thompson S, et al (1991) Demonstration that CFTR is a chloride channel by alteration of its anion selectivity. Science 253:202-205. https://doi.org/10.1126/science.1712984

5. Saint-Criq V, Gray MA (2017) Role of CFTR in epithelial physiology. Cellular and Molecular Life Sciences 74:93-115. https://doi.org/10.1007/s00018-016-2391-y

6. Smith JJ, Travis SM, Greenberg EP, Welsh MJ (1996) Cystic Fibrosis Airway Epithelia Fail to Kill Bacteria Because of Abnormal Airway Surface Fluid. Cell 85:229-236. https://doi.org/10.1016/S0092-8674(00)81099-5

7. Ishiguro H, Steward MC, Naruse S, et al (2009) CFTR Functions as a Bicarbonate Channel in Pancreatic Duct Cells. Journal of General Physiology 133:315-326. https://doi.org/10.1085/jgp.200810122

8. Reddy M, Quinton P (2003) Functional interaction of CFTR and ENaC in sweat glands. Pflügers Archiv - European Journal of Physiology 445:499-503. https://doi.org/10.1007/s00424-002-0959-x

9. $\quad$ Liu Y, Wang D-K, Chen L-M (2012) The Physiology of Bicarbonate Transporters in Mammalian Reproduction1. Biology of Reproduction 86:. https://doi.org/10.1095/biolreprod.111.096826

10. Seidler UE (2013) Gastrointestinal HCO3- transport and epithelial protection in the gut: new techniques, transport pathways and regulatory pathways. Current Opinion in Pharmacology 13:900-908. https://doi.org/10.1016/j.coph.2013.10.001

11. Elborn JS (2016) Cystic fibrosis. The Lancet 388:2519-2531. https://doi.org/10.1016/S0140-6736(16)00576-6

12. Dean M, Annilo T (2005) evolution of the ATP-binding cassette (ABC) transporter superfamily in vertebrates. Annual Review of Genomics and Human Genetics 6:123-142. https://doi.org/10.1146/annurev.genom.6.080604.162122

13. Rees DC, Johnson E, Lewinson O (2009) ABC transporters: the power to change. Nature Reviews Molecular Cell Biology 10:218-227. https://doi.org/10.1038/nrm2646 
14. Locher KP (2016) Mechanistic diversity in ATP-binding cassette (ABC) transporters. Nature Structural \& Molecular Biology 23:487-493.

https://doi.org/10.1038/nsmb.3216

15. Ikuma M, Welsh MJ (2000) Regulation of CFTR Cl- channel gating by ATP binding and hydrolysis. Proceedings of the National Academy of Sciences 97:8675-8680. https://doi.org/10.1073/pnas.140220597

16. Cheng SH, Rich DP, Marshall J, et al (1991) Phosphorylation of the R domain by cAMP-dependent protein kinase regulates the CFTR chloride channel. Cell 66:1027-1036. https://doi.org/10.1016/0092-8674(91)90446-6

17. Mihályi C, lordanov I, Töröcsik B, Csanády L (2020) Simple binding of protein kinase A prior to phosphorylation allows CFTR anion channels to be opened by nucleotides. Proceedings of the National Academy of Sciences 117:21740-21746. https://doi.org/10.1073/pnas.2007910117

18. Zhang Z, Chen J (2016) Atomic Structure of the Cystic Fibrosis Transmembrane Conductance Regulator. Cell 167:1586-1597.e9.

https://doi.org/10.1016/j.cell.2016.11.014

19. Liu F, Zhang Z, Csanády L, et al (2017) Molecular Structure of the Human CFTR Ion Channel. Cell 169:85-95.e8. https://doi.org/10.1016/j.cell.2017.02.024

20. Zhang Z, Liu F, Chen J (2017) Conformational Changes of CFTR upon Phosphorylation and ATP Binding. Cell 170:483-491.e8. https://doi.org/10.1016/j.cell.2017.06.041

21. Zhang Z, Liu F, Chen J (2018) Molecular structure of the ATP-bound, phosphorylated human CFTR. Proceedings of the National Academy of Sciences 115:12757-12762. https://doi.org/10.1073/pnas.1815287115

22. Liu F, Zhang Z, Levit A, et al (2019) Structural identification of a hotspot on CFTR for potentiation. Science 364:1184-1188. https://doi.org/10.1126/science.aaw7611

23. Vergani P, Lockless SW, Nairn AC, Gadsby DC (2005) CFTR channel opening by ATP-driven tight dimerization of its nucleotide-binding domains. Nature 433:876880. https://doi.org/10.1038/nature03313

24. Mihályi C, Töröcsik B, Csanády L (2016) Obligate coupling of CFTR pore opening to tight nucleotide-binding domain dimerization. eLife 5:.

https://doi.org/10.7554/eLife.18164

25. Jih K-Y, Sohma Y, Hwang T-C (2012) Nonintegral stoichiometry in CFTR gating revealed by a pore-lining mutation. Journal of General Physiology 140:347-359. https://doi.org/10.1085/jgp.201210834

26. Dawson RJP, Locher KP (2006) Structure of a bacterial multidrug ABC transporter. Nature 443:180-185. https://doi.org/10.1038/nature05155 
27. Gadsby DC (2009) lon channels versus ion pumps: the principal difference, in principle. Nature Reviews Molecular Cell Biology 10:344-352.

https://doi.org/10.1038/nrm2668

28. Gao X, Hwang T-C (2015) Localizing a gate in CFTR. Proceedings of the National Academy of Sciences 112:2461-2466. https://doi.org/10.1073/pnas.1420676112

29. SHEPPARD DN, WELSH MJ (1999) Structure and Function of the CFTR Chloride Channel. Physiological Reviews 79:S23-S45.

https://doi.org/10.1152/physrev.1999.79.1.S23

30. Corradi V, Gu R-X, Vergani P, Tieleman DP (2018) Structure of Transmembrane Helix 8 and Possible Membrane Defects in CFTR. Biophysical Journal 114:17511754. https://doi.org/10.1016/j.bpj.2018.03.003

31. Farkas B, Tordai H, Padányi $R$, et al (2020) Discovering the chloride pathway in the CFTR channel. Cellular and Molecular Life Sciences 77:765-778. https://doi.org/10.1007/s00018-019-03211-4

32. Hoffmann B, Elbahnsi A, Lehn P, et al (2018) Combining theoretical and experimental data to decipher CFTR 3D structures and functions. Cellular and Molecular Life Sciences 75:3829-3855. https://doi.org/10.1007/s00018-018-28357

33. Li M-S, Cowley EA, el Hiani Y, Linsdell P (2018) Functional organization of cytoplasmic portals controlling access to the cystic fibrosis transmembrane conductance regulator (CFTR) chloride channel pore. Journal of Biological Chemistry 293:5649-5658. https://doi.org/10.1074/jbc.RA117.001373

34. el Hiani Y, Linsdell P (2015) Functional Architecture of the Cytoplasmic Entrance to the Cystic Fibrosis Transmembrane Conductance Regulator Chloride Channel Pore. Journal of Biological Chemistry 290:15855-15865. https://doi.org/10.1074/jbc.M115.656181

35. Rao S, Klesse G, Lynch Cl, et al (2021) Molecular Simulations of Hydrophobic Gating of Pentameric Ligand Gated Ion Channels: Insights into Water and Ions. The Journal of Physical Chemistry B 125:981-994. https://doi.org/10.1021/acs.jpcb.0c09285

36. Jensen MO, Borhani DW, Lindorff-Larsen K, et al (2010) Principles of conduction and hydrophobic gating in $\mathrm{K}+$ channels. Proceedings of the National Academy of Sciences 107:5833-5838. https://doi.org/10.1073/pnas.0911691107

37. Sotomayor M, Schulten K (2004) Molecular Dynamics Study of Gating in the Mechanosensitive Channel of Small Conductance MscS. Biophysical Journal 87:3050-3065. https://doi.org/10.1529/biophysj.104.046045

38. Rahman KS, Cui G, Harvey SC, McCarty NA (2013) Modeling the Conformational Changes Underlying Channel Opening in CFTR. PLoS ONE 8:e74574. https://doi.org/10.1371/journal.pone.0074574 
39. Corradi V, Vergani P, Tieleman DP (2015) Cystic Fibrosis Transmembrane Conductance Regulator (CFTR). Journal of Biological Chemistry 290:2289122906. https://doi.org/10.1074/jbc.M115.665125

40. Dalton J, Kalid O, Schushan M, et al (2012) New Model of Cystic Fibrosis Transmembrane Conductance Regulator Proposes Active Channel-like Conformation. Journal of Chemical Information and Modeling 52:1842-1853. https://doi.org/10.1021/ci2005884

41. Negoda A, Hogan MS, Cowley EA, Linsdell P (2019) Contribution of the eighth transmembrane segment to the function of the CFTR chloride channel pore.

Cellular and Molecular Life Sciences 76:2411-2423.

https://doi.org/10.1007/s00018-019-03043-2

42. Fay JF, Aleksandrov LA, Jensen TJ, et al (2018) Cryo-EM Visualization of an Active High Open Probability CFTR Anion Channel. Biochemistry 57:6234-6246. https://doi.org/10.1021/acs.biochem.8b00763

43. Hwang T-C, Yeh J-T, Zhang J, et al (2018) Structural mechanisms of CFTR function and dysfunction. Journal of General Physiology 150:539-570. https://doi.org/10.1085/jgp.201711946

44. Linsdell $P(2021)$ On the relationship between anion binding and chloride conductance in the CFTR anion channel. Biochimica et Biophysica Acta (BBA) Biomembranes 1863:183558. https://doi.org/10.1016/j.bbamem.2021.183558

45. Linsdell P (2005) Location of a Common Inhibitor Binding Site in the Cytoplasmic Vestibule of the Cystic Fibrosis Transmembrane Conductance Regulator Chloride Channel Pore. Journal of Biological Chemistry 280:8945-8950.

https://doi.org/10.1074/jbc.M414354200

46. st. Aubin CN, Zhou J-J, Linsdell P (2007) Identification of a Second Blocker Binding Site at the Cytoplasmic Mouth of the Cystic Fibrosis Transmembrane Conductance Regulator Chloride Channel Pore. Molecular Pharmacology 71:1360-1368. https://doi.org/10.1124/mol.106.031732

47. Ge N, Muise CN, Gong X, Linsdell P (2004) Direct Comparison of the Functional Roles Played by Different Transmembrane Regions in the Cystic Fibrosis Transmembrane Conductance Regulator Chloride Channel Pore. Journal of Biological Chemistry 279:55283-55289. https://doi.org/10.1074/jbc.M411935200

48. Aubin CN st., Linsdell P (2006) Positive Charges at the Intracellular Mouth of the Pore Regulate Anion Conduction in the CFTR Chloride Channel. Journal of General Physiology 128:535-545. https://doi.org/10.1085/jgp.200609516

49. el Hiani Y, Linsdell P (2012) Tuning of CFTR Chloride Channel Function by Location of Positive Charges within the Pore. Biophysical Journal 103:1719-1726. https://doi.org/10.1016/j.bpj.2012.09.020

50. Zhou J-J, Li M-S, Qi J, Linsdell P (2010) Regulation of conductance by the number of fixed positive charges in the intracellular vestibule of the CFTR chloride channel 
pore. Journal of General Physiology 135:229-245.

https://doi.org/10.1085/jgp.200910327

51. Linsdell P, Negoda A, Cowley EA, el Hiani Y (2020) Electrostatic Tuning of Anion Attraction from the Cytoplasm to the Pore of the CFTR Chloride Channel. Cell Biochemistry and Biophysics 78:15-22. https://doi.org/10.1007/s12013-019-00899w

52. Linsdell P (2017) Architecture and functional properties of the CFTR channel pore. Cellular and Molecular Life Sciences 74:67-83. https://doi.org/10.1007/s00018016-2389-5

53. McDonough S, Davidson N, Lester HA, McCarty NA (1994) Novel pore-lining residues in CFTR that govern permeation and open-channel block. Neuron 13:623-634. https://doi.org/10.1016/0896-6273(94)90030-2

54. Gao X, Hwang T-C (2016) Spatial positioning of CFTR's pore-lining residues affirms an asymmetrical contribution of transmembrane segments to the anion permeation pathway. Journal of General Physiology 147:407-422. https://doi.org/10.1085/jgp.201511557

55. Negoda A, el Hiani Y, Cowley EA, Linsdell P (2017) Contribution of a leucine residue in the first transmembrane segment to the selectivity filter region in the CFTR chloride channel. Biochimica et Biophysica Acta (BBA) - Biomembranes 1859:1049-1058. https://doi.org/10.1016/j.bbamem.2017.02.014

56. Linsdell P (2001) Relationship between anion binding and anion permeability revealed by mutagenesis within the cystic fibrosis transmembrane conductance regulator chloride channel pore. The Journal of Physiology 531:51-66. https://doi.org/10.1111/j.1469-7793.2001.0051j.x

57. Linsdell P, Zheng S-X, Hanrahan JW (1998) Non-pore lining amino acid side chains influence anion selectivity of the human CFTR $\mathrm{Cl}-$ channel expressed in mammalian cell lines. The Journal of Physiology 512:1-16. https://doi.org/10.1111/j.1469-7793.1998.001bf.x

58. Negoda A, Cowley EA, el Hiani Y, Linsdell P (2018) Conformational change of the extracellular parts of the CFTR protein during channel gating. Cellular and Molecular Life Sciences 75:3027-3038. https://doi.org/10.1007/s00018-018-27770

59. Sheppard DN, Travis SM, Ishihara H, Welsh MJ (1996) Contribution of Proline Residues in the Membrane-spanning Domains of Cystic Fibrosis Transmembrane Conductance Regulator to Chloride Channel Function. Journal of Biological Chemistry 271:14995-15001. https://doi.org/10.1074/jbc.271.25.14995

60. Smith SS, Steinle ED, Meyerhoff ME, Dawson DC (1999) Cystic Fibrosis Transmembrane Conductance Regulator. Journal of General Physiology 114:799818. https://doi.org/10.1085/jgp.114.6.799

61. Linsdell P, Evagelidis A, Hanrahan JW (2000) Molecular Determinants of Anion Selectivity in the Cystic Fibrosis Transmembrane Conductance Regulator Chloride 
Channel Pore. Biophysical Journal 78:2973-2982. https://doi.org/10.1016/S00063495(00)76836-6

62. Smith SS, Liu X, Zhang Z-R, et al (2001) Cftr: Covalent and noncovalent modification suggests a role for fixed charges in anion conduction. Journal of General Physiology 118:407-432. https://doi.org/10.1085/jgp.118.4.407

63. Zhou J-J, Fatehi M, Linsdell P (2008) Identification of positive charges situated at the outer mouth of the CFTR chloride channel pore. Pflügers Archiv - European Journal of Physiology 457:351-360. https://doi.org/10.1007/s00424-008-0521-6

64. Fatehi M, Linsdell P (2009) Novel Residues Lining the CFTR Chloride Channel Pore Identified by Functional Modification of Introduced Cysteines. Journal of Membrane Biology 228:151-164. https://doi.org/10.1007/s00232-009-9167-3

65. Dzubiella J, Allen RJ, Hansen J-P (2004) Electric field-controlled water permeation coupled to ion transport through a nanopore. The Journal of Chemical Physics 120:5001-5004. https://doi.org/10.1063/1.1665656

66. Klesse G, Tucker SJ, Sansom MSP (2020) Electric Field Induced Wetting of a Hydrophobic Gate in a Model Nanopore Based on the 5-HT 3 Receptor Channel. ACS Nano 14:10480-10491. https://doi.org/10.1021/acsnano.0c04387

67. Rao S, Lynch Cl, Klesse G, et al (2018) Water and hydrophobic gates in ion channels and nanopores. Faraday Discussions 209:231-247. https://doi.org/10.1039/C8FD00013A

68. Aryal P, Sansom MSP, Tucker SJ (2015) Hydrophobic Gating in Ion Channels. Journal of Molecular Biology 427:121-130.

https://doi.org/10.1016/j.jmb.2014.07.030

69. Lomize MA, Pogozheva ID, Joo H, et al (2012) OPM database and PPM web server: resources for positioning of proteins in membranes. Nucleic Acids Research 40:D370-D376. https://doi.org/10.1093/nar/gkr703

70. Jo S, Kim T, lyer VG, Im W (2008) CHARMM-GUI: A web-based graphical user interface for CHARMM. Journal of Computational Chemistry 29:1859-1865. https://doi.org/10.1002/jcc.20945

71. Kim S, Lee J, Jo S, et al (2017) CHARMM-GUI ligand reader and modeler for CHARMM force field generation of small molecules. Journal of Computational Chemistry 38:1879-1886. https://doi.org/10.1002/jcc.24829

72. Abraham MJ, Murtola T, Schulz R, et al (2015) GROMACS: High performance molecular simulations through multi-level parallelism from laptops to supercomputers. SoftwareX 1-2:19-25. https://doi.org/10.1016/j.softx.2015.06.001

73. Best RB, Zhu X, Shim J, et al (2012) Optimization of the Additive CHARMM AllAtom Protein Force Field Targeting Improved Sampling of the Backbone $\phi, \psi$ and Side-Chain $X_{1}$ and $X_{2}$ Dihedral Angles. Journal of Chemical Theory and Computation 8:3257-3273. https://doi.org/10.1021/ct300400x 
74. Huang J, MacKerell AD (2013) CHARMM36 all-atom additive protein force field: Validation based on comparison to NMR data. Journal of Computational Chemistry 34:2135-2145. https://doi.org/10.1002/jcc.23354

75. Klauda JB, Venable RM, Freites JA, et al (2010) Update of the CHARMM All-Atom Additive Force Field for Lipids: Validation on Six Lipid Types. The Journal of Physical Chemistry B 114:7830-7843. https://doi.org/10.1021/jp101759q

76. Jorgensen WL, Chandrasekhar J, Madura JD, et al (1983) Comparison of simple potential functions for simulating liquid water. The Journal of Chemical Physics 79:926-935. https://doi.org/10.1063/1.445869

77. Nosé S (1984) A molecular dynamics method for simulations in the canonical ensemble. Molecular Physics 52:255-268.

https://doi.org/10.1080/00268978400101201

78. Hoover WG (1985) Canonical dynamics: Equilibrium phase-space distributions. Physical Review A 31:1695-1697. https://doi.org/10.1103/PhysRevA.31.1695

79. Parrinello M, Rahman A (1980) Crystal Structure and Pair Potentials: A MolecularDynamics Study. Physical Review Letters 45:1196-1199.

https://doi.org/10.1103/PhysRevLett.45.1196

80. Nosé S, Klein ML (1983) Constant pressure molecular dynamics for molecular systems. Molecular Physics 50:1055-1076.

https://doi.org/10.1080/00268978300102851

81. Verlet $L$ (1967) Computer "Experiments" on Classical Fluids. I. Thermodynamical Properties of Lennard-Jones Molecules. Physical Review 159:98-103. https://doi.org/10.1103/PhysRev.159.98

82. Páll S, Hess B (2013) A flexible algorithm for calculating pair interactions on SIMD architectures. Computer Physics Communications 184:2641-2650.

https://doi.org/10.1016/j.cpc.2013.06.003

83. Darden T, York D, Pedersen L (1993) Particle mesh Ewald: An N ·log( N ) method for Ewald sums in large systems. The Journal of Chemical Physics 98:1008910092. https://doi.org/10.1063/1.464397

84. Essmann U, Perera L, Berkowitz ML, et al (1995) A smooth particle mesh Ewald method. The Journal of Chemical Physics 103:8577-8593.

https://doi.org/10.1063/1.470117

85. Hess B (2008) P-LINCS: A Parallel Linear Constraint Solver for Molecular Simulation. Journal of Chemical Theory and Computation 4:116-122. https://doi.org/10.1021/ct700200b

86. Roux B (2008) The Membrane Potential and its Representation by a Constant Electric Field in Computer Simulations. Biophysical Journal 95:4205-4216. https://doi.org/10.1529/biophysj.108.136499 
87. Gumbart J, Khalili-Araghi F, Sotomayor M, Roux B (2012) Constant electric field simulations of the membrane potential illustrated with simple systems. Biochimica et Biophysica Acta (BBA) - Biomembranes 1818:294-302.

https://doi.org/10.1016/j.bbamem.2011.09.030

88. Humphrey W, Dalke A, Schulten K (1996) VMD: Visual molecular dynamics. Journal of Molecular Graphics 14:33-38. https://doi.org/10.1016/02637855(96)00018-5

89. McGibbon RT, Beauchamp KA, Harrigan MP, et al (2015) MDTraj: A Modern Open Library for the Analysis of Molecular Dynamics Trajectories. Biophysical Journal 109:1528-1532. https://doi.org/10.1016/j.bpj.2015.08.015

90. Sehnal D, Svobodová Vařeková R, Berka K, et al (2013) MOLE 2.0: advanced approach for analysis of biomacromolecular channels. Journal of Cheminformatics 5:39. https://doi.org/10.1186/1758-2946-5-39 


\section{Figures}
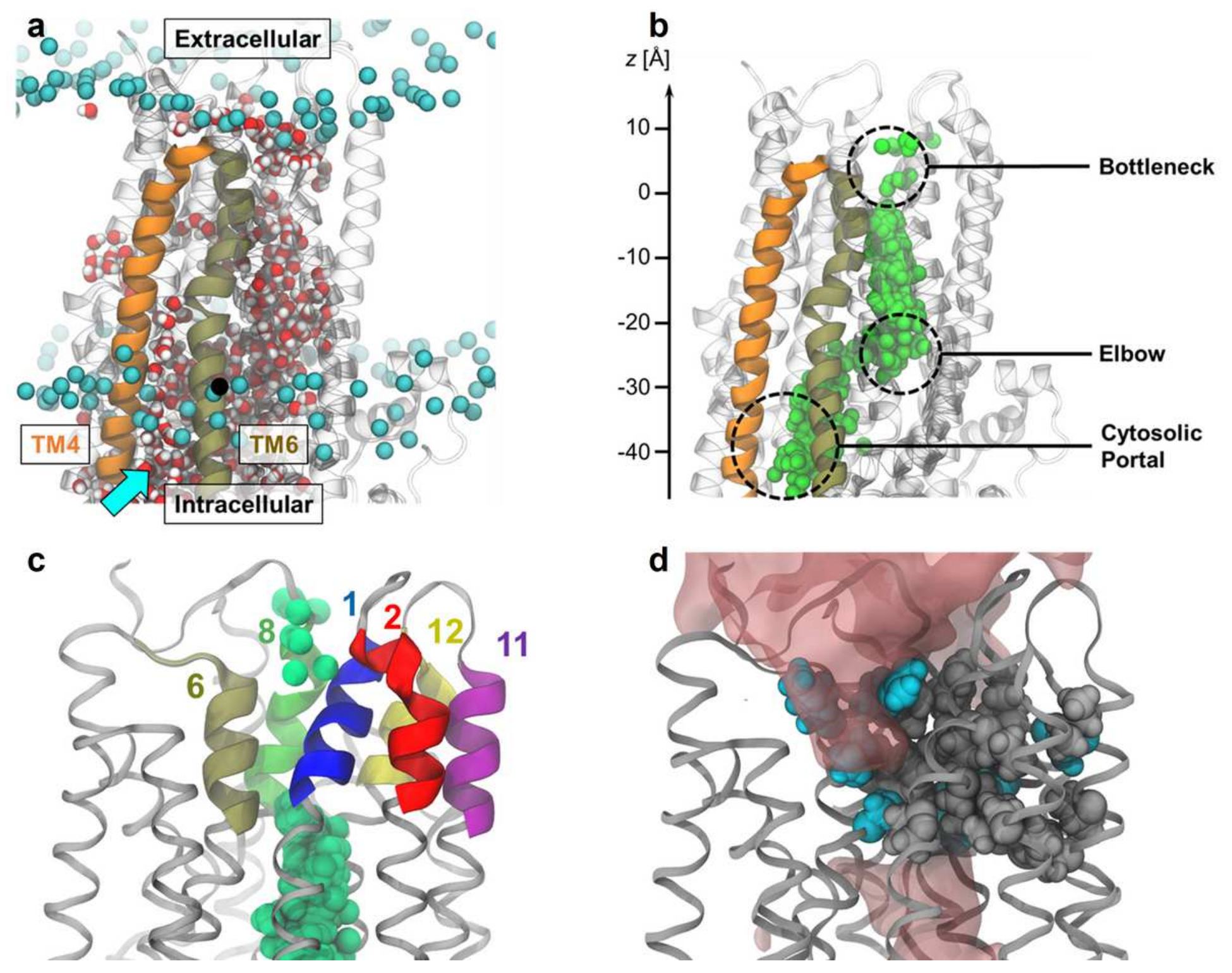

Figure 1

Overview of the CFTR channel pore. (A) Simulation snapshot showing the waterfilled channel cavity (inner vestibule). The inner vestibule is accessible to water from the intracellular space through the "cytosolic portal" (cyan arrow) formed by TM helices 4 and 6. Phosphorus atoms (cyan spheres) of POPC headgroups indicate membrane position. Bulk water and lipid tails are omitted for clarity. (B) Spatial view of occupancy of $\mathrm{Cl}$ - ions (green spheres) inside the channel cavity from many timesteps overlaid. (C) Cartoon representation of TM helix segments lining the bottleneck region of the pore together with $\mathrm{Cl}$ ions drawn from multiple timesteps. (D) The bottleneck region of the pore is lined by many hydrophobic residues (grey), disfavouring the presence of water (red surfaces). Selected hydrophilic/charged residues (cyan) are also shown. 

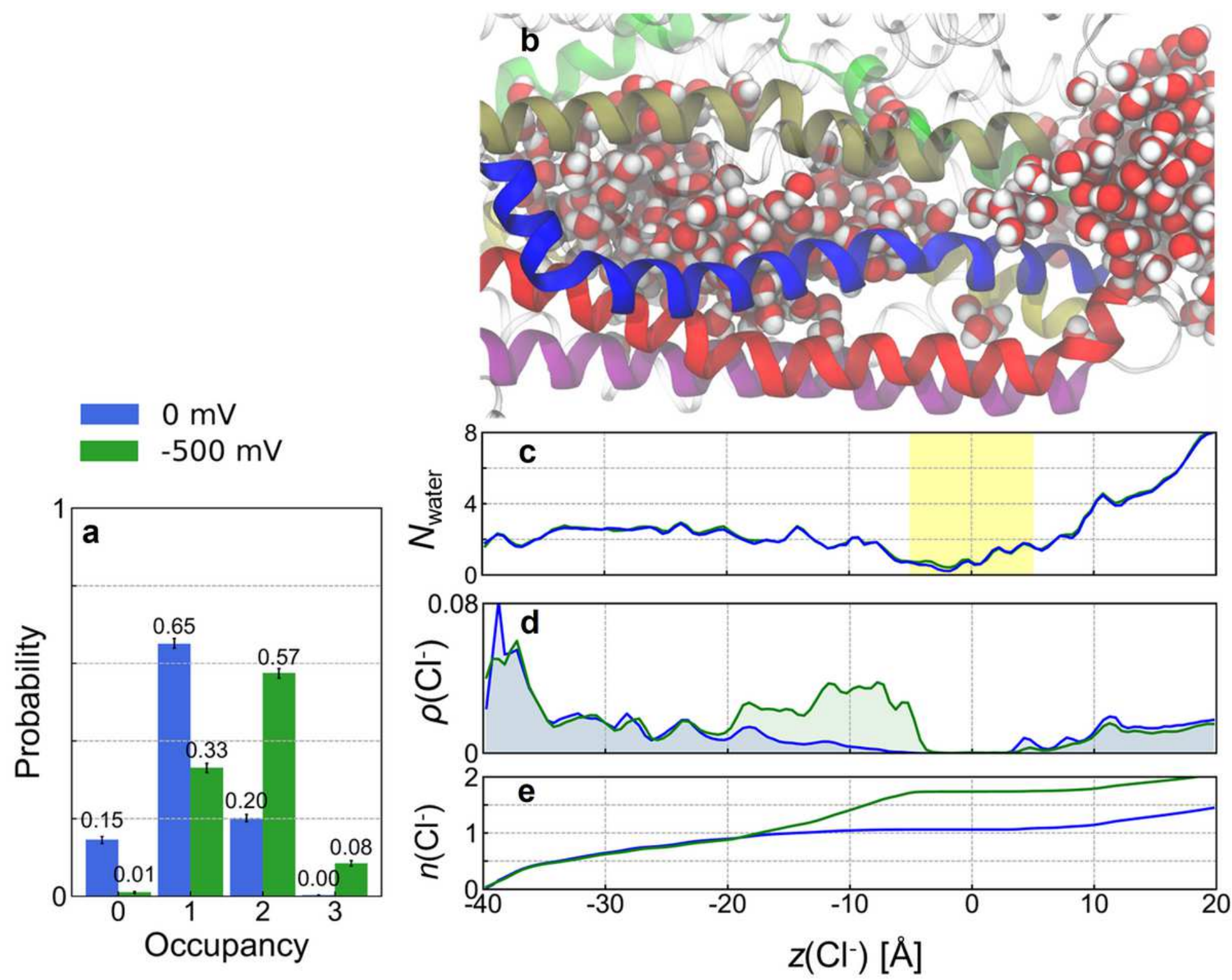

Figure 2

Effect of transmembrane voltage on water and Cl- occupancy of the CFTR channel. (A) Probability distribution of the number of $\mathrm{Cl}$ - ions occupying the inner vestibule (blue) without and (green) with applied voltage. Error bars represent bootstrapped 99\% confidence intervals. (B) Snapshot of the CFTR channel filled with water molecules used as positional reference for (C)-(E). (C) Average number density of water molecules (Nwater) in the channel vestibule computed along the z-axis. The bottleneck region is highlighted in yellow shading. (D) Average population density of Clions, $\rho(\mathrm{Cl}-)$, inside the channel pore (blue) without and (green) with $-500 \mathrm{mV}$ transmembrane voltage. The $\mathrm{Cl}$ - occupancy of the region $-20<\mathrm{Z}$ $<0 \AA$ is enhanced by voltage. (E) Cumulative population densities of $\mathrm{Cl}$ - ions, $\mathrm{n}(\mathrm{Cl}-)$, inside the channel pore computed by integrating $\rho(\mathrm{Cl}-)$ from (D). 


\section{a}
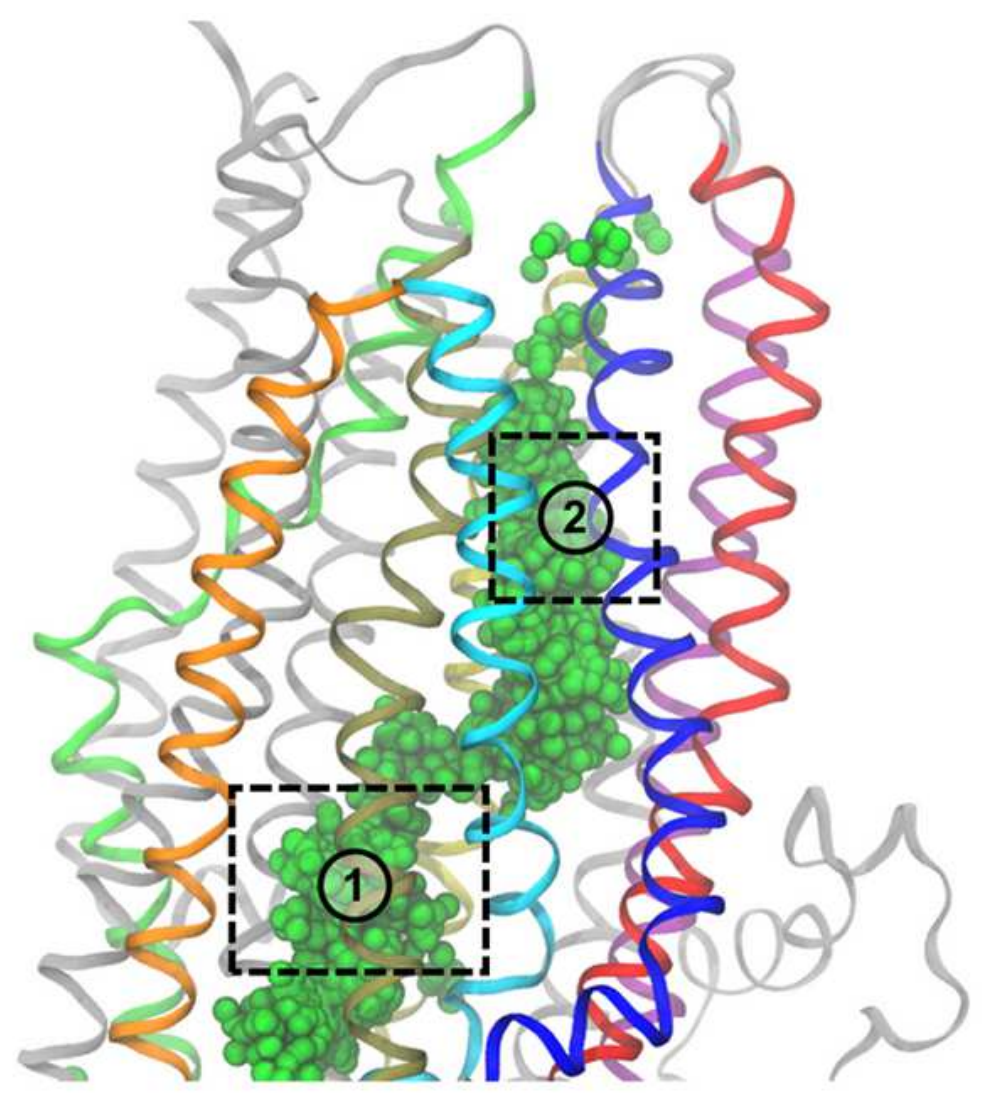
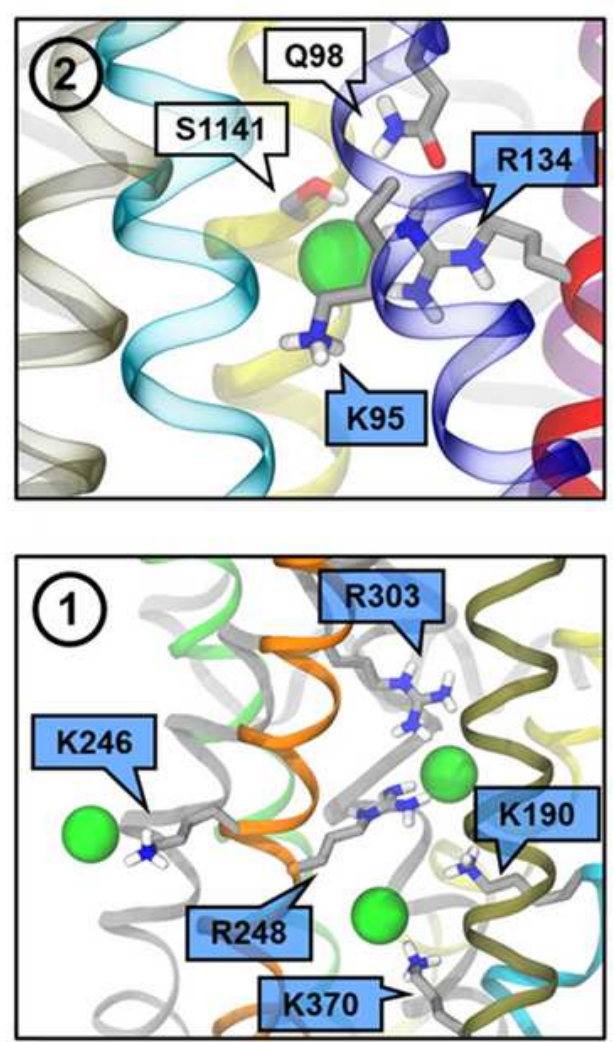

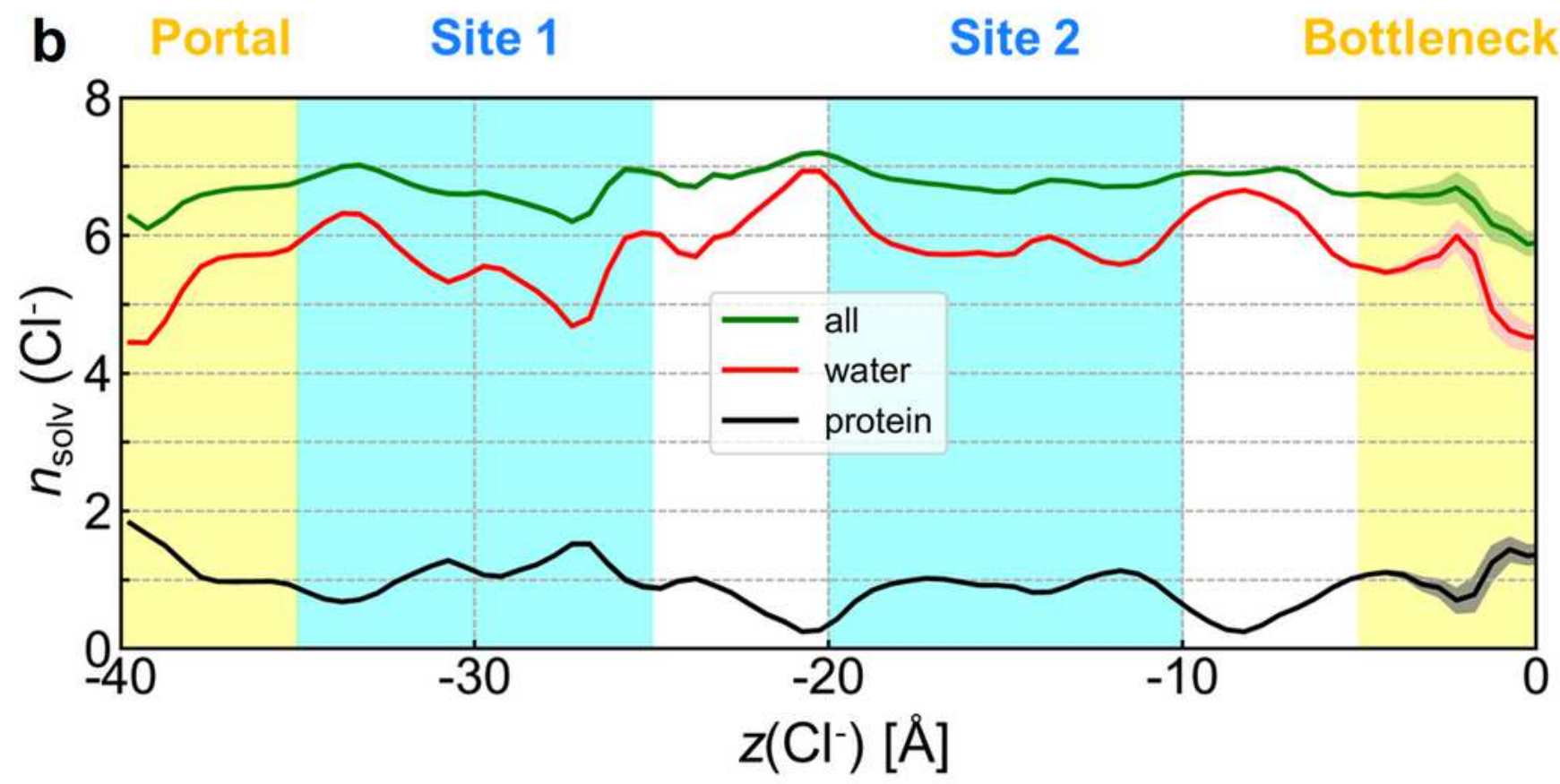

Figure 3

Chloride solvation in the inner vestibule. (A) Left: overview of the $\mathrm{Cl}$-permeation pathway with overlaid ions (green spheres) from simulations in the presence of voltage with TM helices 1 (blue), 2 (red), 3 (cyan), 4 (orange), 6 (tan), 8 (lime), 11 (purple), and 12 (yellow). The locations of two Cl- binding sites 1 and 2 are indicated in dashed boxes. Right: close-up views of binding sites 1 and 2. Amino acid sidechains in direct contact with $\mathrm{Cl}$ - ions (green spheres) are shown. The labels of positively charged 
residues, including K246 and K370 from the cytosolic portal, are shaded in indigo. (B) Coordination of Clions inside the channel cavity from simulations with voltage. The average numbers of protein residues (black) and water molecules (red) in the first solvation shell of $\mathrm{Cl}$-ions (nsolv) along the intracellular pathway are shown together with their sum (green). Shading represents $99 \%$ bootstrapped confidence intervals. Error bars are largest in the bottleneck region, which is least visited.

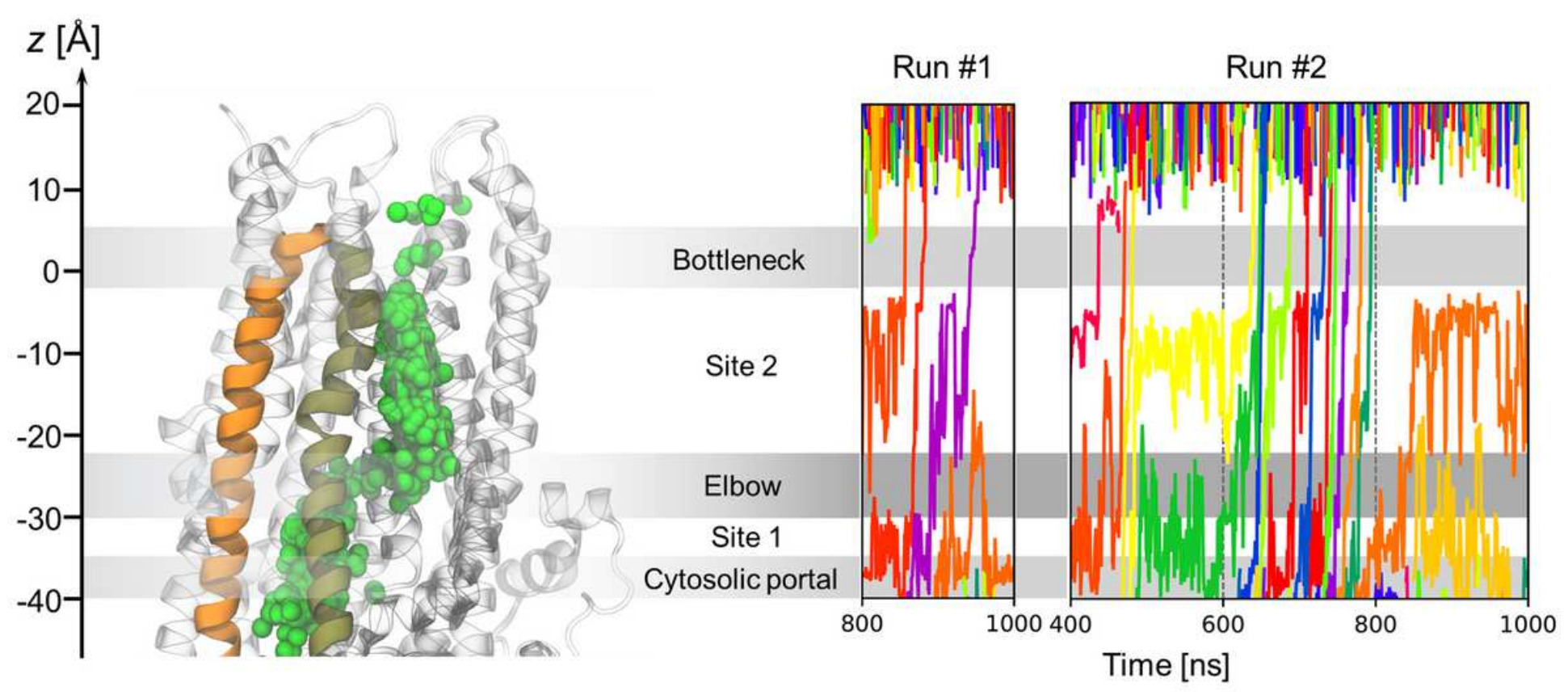

Figure 4

Spontaneous permeation of $\mathrm{Cl}$ - ions in the CFTR channel under applied voltage. Left: overview of the Clpermeation pathway with overlaid ions (green spheres) from simulations in presence of voltage highlighting TM helices 4 (orange) and 6 (tan). Right: time series of axial $\mathrm{Cl}$ - ion positions from the two simulation runs in which permeation occurred $(-500 \mathrm{mV})$. lon permeation events are indicated by traces passing through the bottleneck region. Individual $\mathrm{Cl}$ - ions are distinguished by color for clarity. 

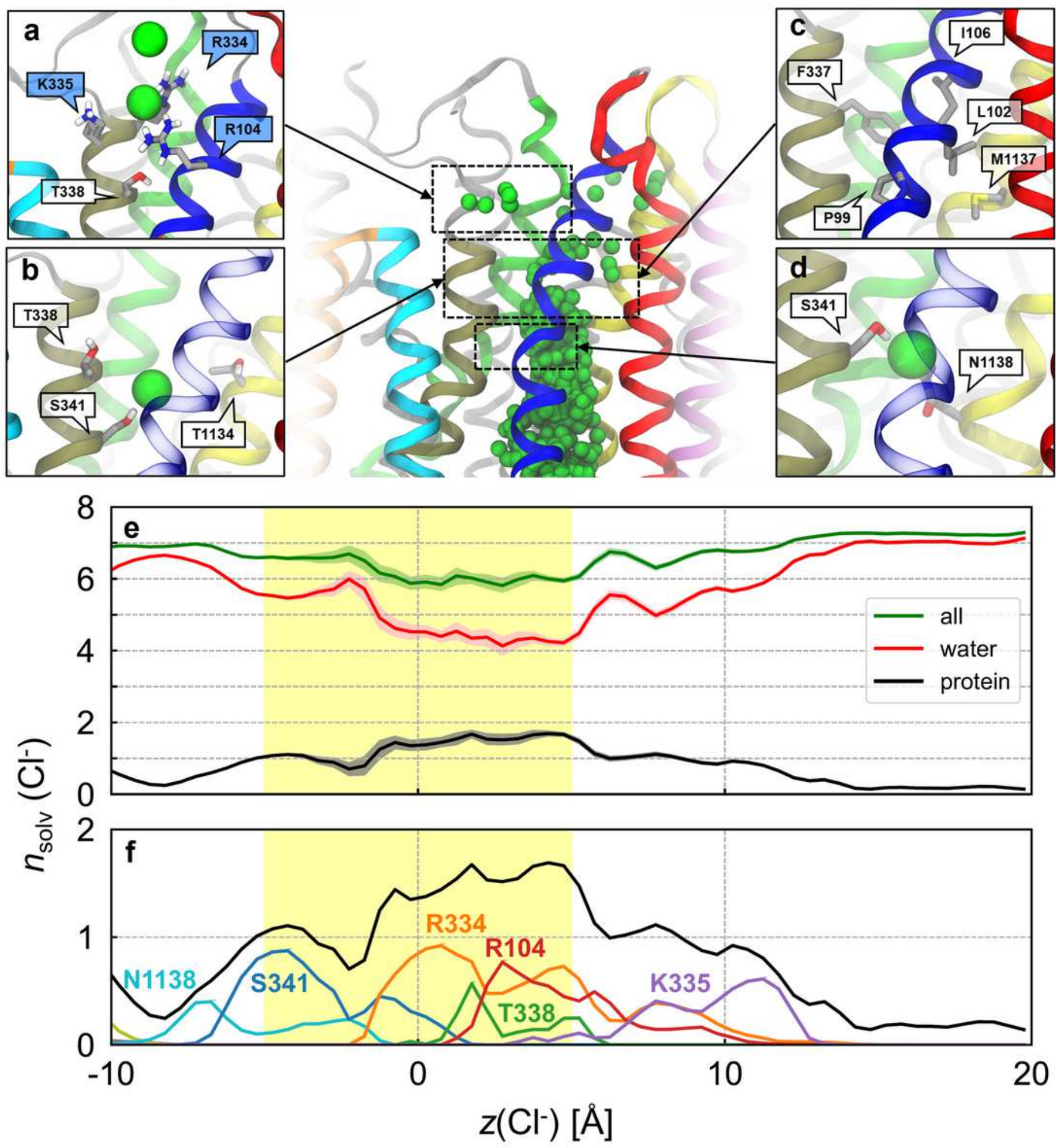

Figure 5

Chloride translocation pathway through the bottleneck region. Top center: overview of the Cl- permeation pathway with overlaid ions (green spheres) from simulations in the presence of voltage with TM helices 1 (blue), 2 (red), 3 (cyan), 4 (orange), 6 (tan), 8 (lime), 11 (purple), and 12 (yellow). (A)-(D) Close-up views showing the residues interacting with permeating $\mathrm{Cl}$ - ions. The labels of positively charged residues are shaded in indigo. (E) Coordination of $\mathrm{Cl}$ - ions inside the channel cavity from simulations with voltage. 
The average numbers of protein residues (black) and water molecules (red) in the first solvation shell of $\mathrm{Cl}$ - ions (nsolv) along the intracellular pathway are shown together with their sum (green). Shading represents $99 \%$ bootstrapped confidence intervals. Chloride ions are less hydrated in the bottleneck region (yellow shading). (F) Significant amino-acid contributions (various colours) to the protein solvation number nsolv (black).
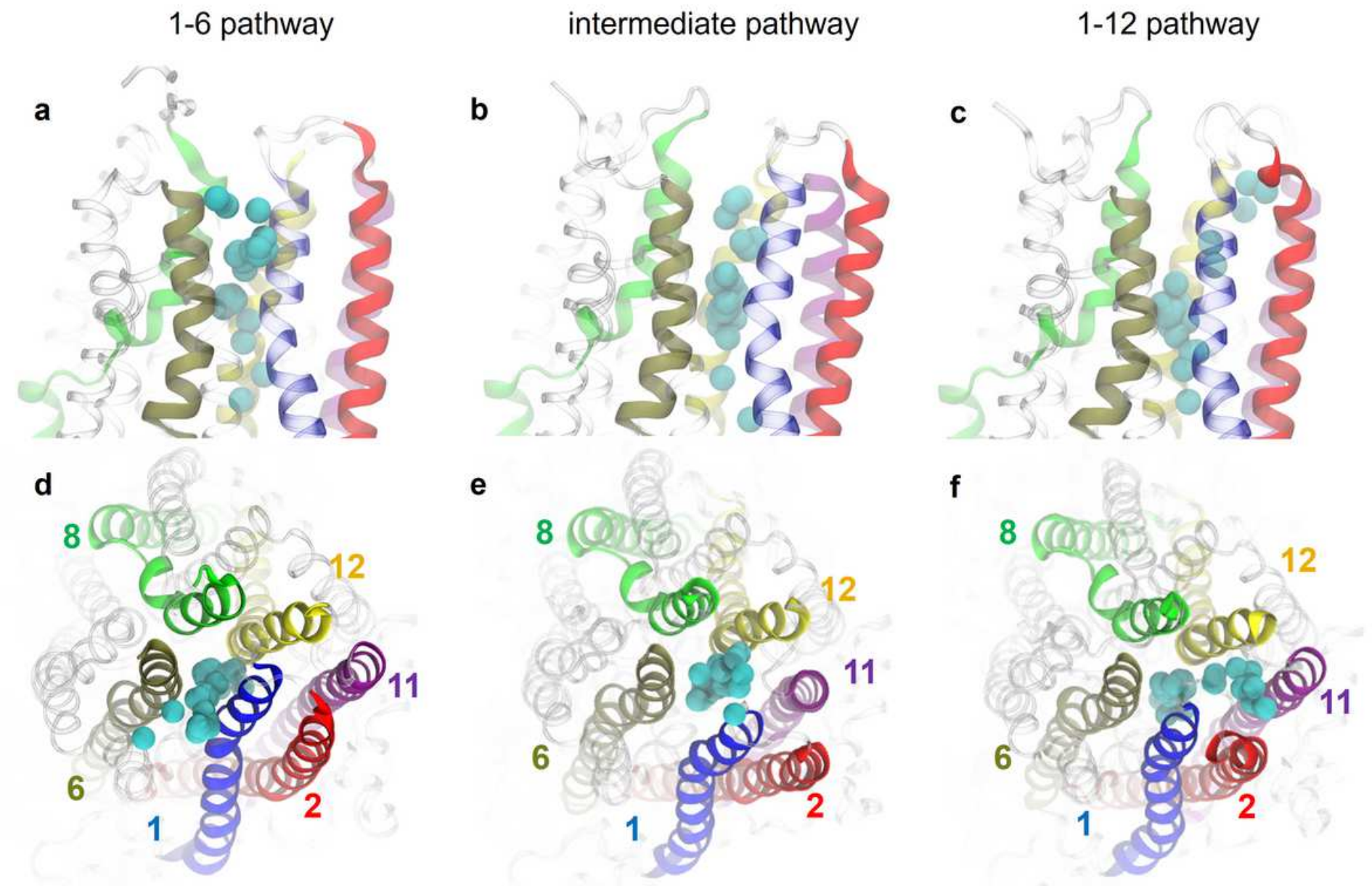

\section{Figure 6}

The three different pathways of $\mathrm{Cl}$ - permeation through the extracellular bottleneck. (Top row) Side views and (bottom row) top views from extracellular space. Pore-lining helices are colour-coded as indicated. Chloride ions (cyan) overlaid from timesteps during translocation. (A), (D) 1-6 pathway; (B), (E) intermediate pathway; (C), (F) 1-12 pathway. 


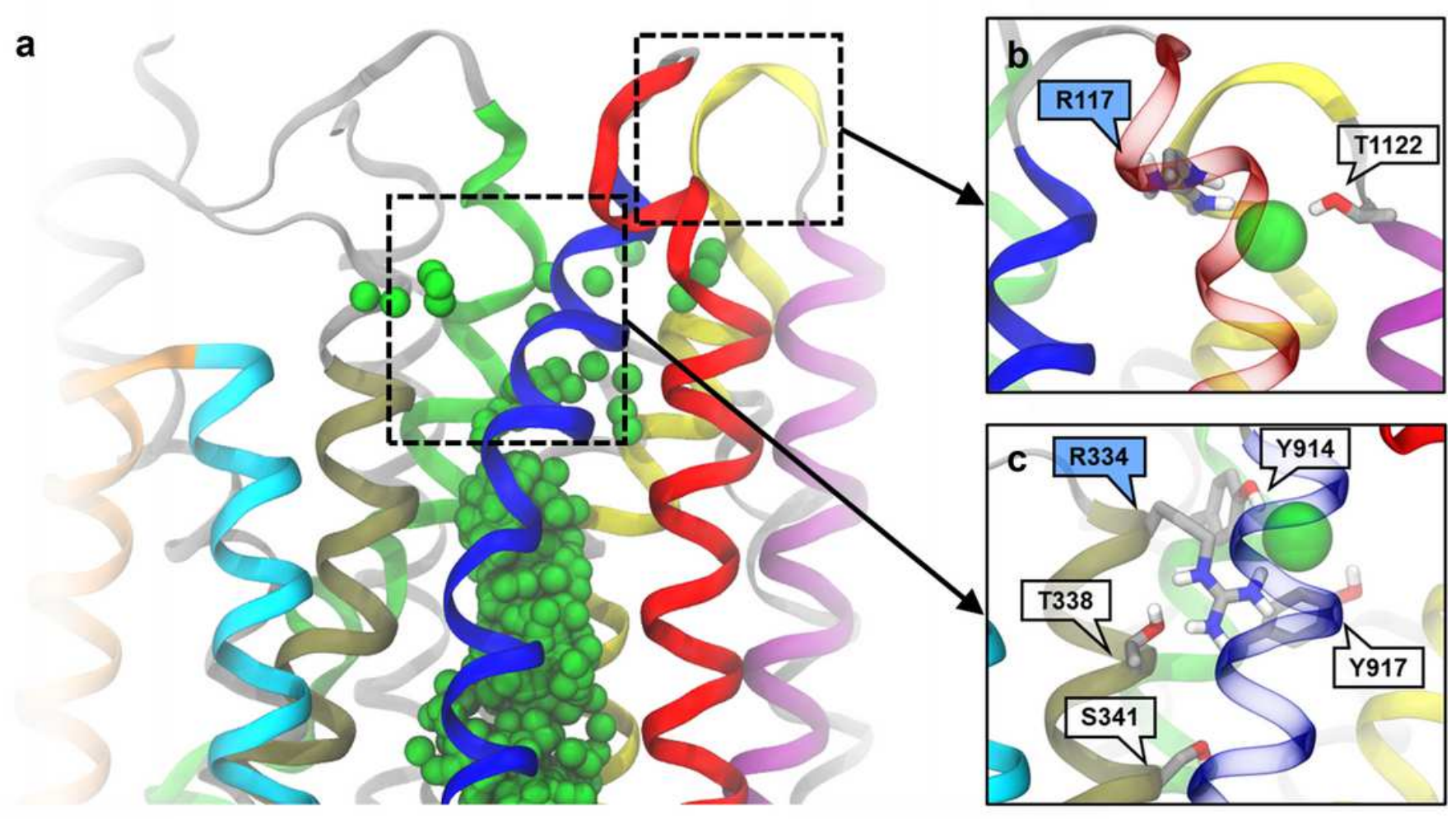

Figure 7

Outer $\mathrm{Cl}$ - binding sites at the extracellular mouth. Boxed regions in the overview of the pore $(\mathrm{A})$ are expanded in insets showing $\mathrm{Cl}$ - exit binding sites for (B) the 1-12 pathway and (C) the intermediate pathway. TM helices 1 (blue), 2 (red), 3 (cyan), 4 (orange), 6 (tan), 8 (lime), 11 (purple), and 12 (yellow) are highlighted. Residue sidechains interacting with $\mathrm{Cl}$ - ions are shown.
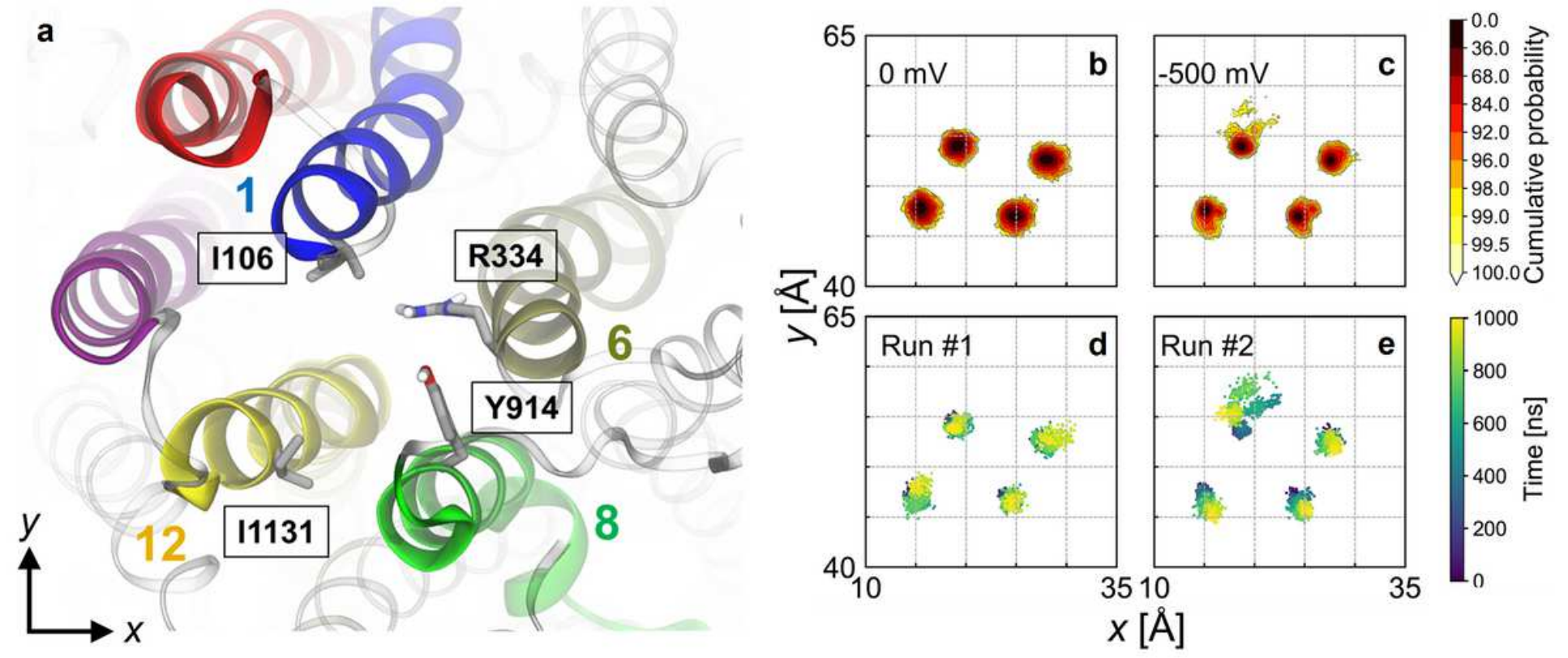

Figure 8 
Structural fluctuations of TM helices lining the extracellular bottleneck region. (A) Top view of the pore from the extracellular side highlighting amino acid residues of the pore-lining helices used to track TM helix movements. (B)-(C) Distributions of xypositions of the extracellular ends of TM helices 1, 6, 8, and 12 in the absence and presence of transmembrane voltage. (D)-(E) Time evolution of TM helices 1, 6, 8, and 12 in conductive simulation runs \#1 and $2(-500 \mathrm{mV})$. In run \#1, a brief departure of TM6 away from the other helices is seen towards $1000 \mathrm{~ns}$. In run \#2, TM1 shows significant fluctuations of up to $\sim 5 \AA$ from its average location.

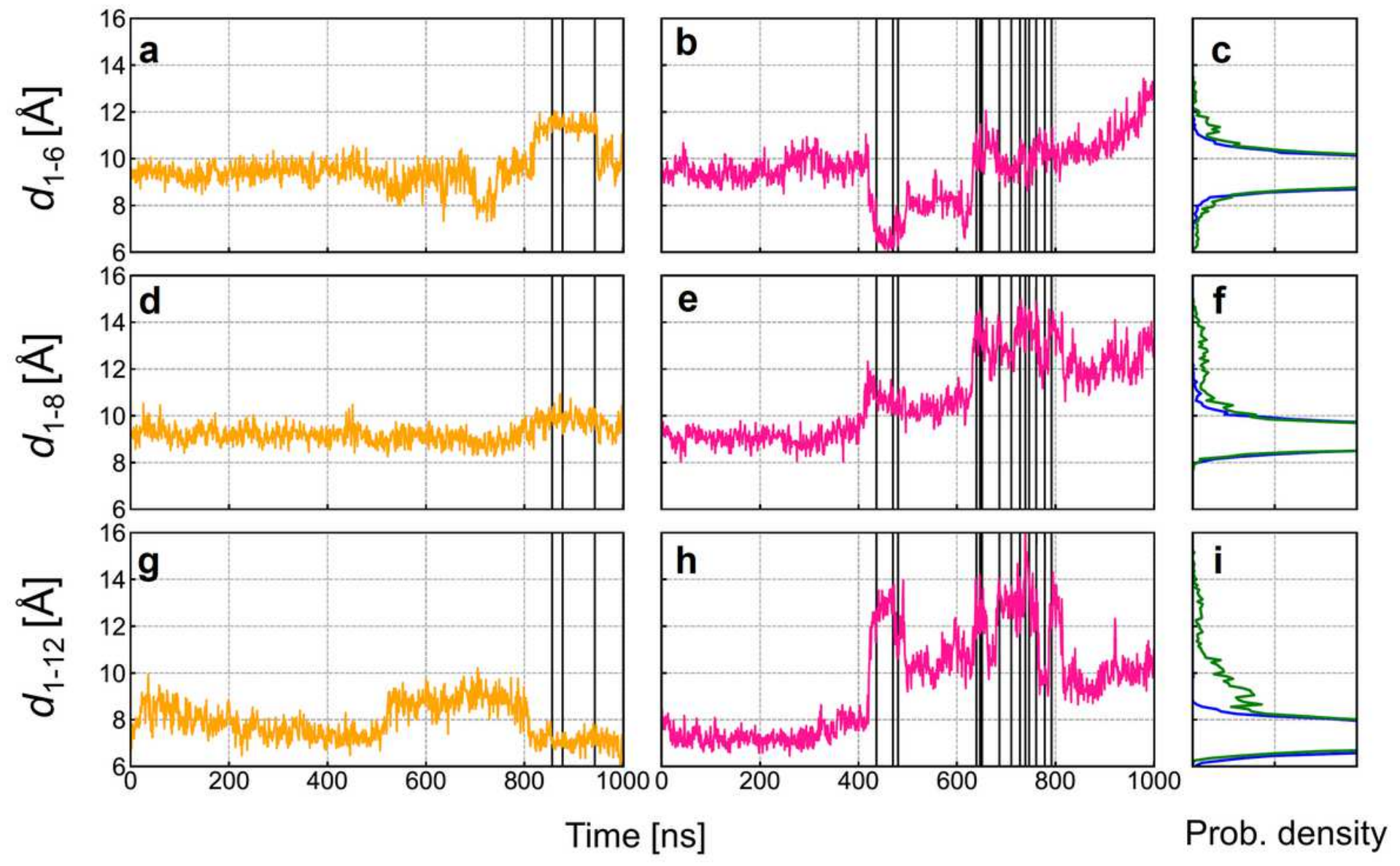

Figure 9

Time evolution of interhelical distances between TM 1 and other pore-lining helices at the extracellular end. Interhelical distances are computed as the distances between $\mathrm{Ca}$ atom pairs of the following residues: d1-6: I106-R334 (top row); d1-8: I106- Y914 (middle row); and d1-12: I106-11131 (bottom row). (A), (D), (G) Time series of interhelical distances for run \#1 (orange traces); (B), (E), (H) same for \#2 (pink traces). Chloride translocation events (see Fig. 4) are indicated as vertical lines. (C), (F), (I) Distributions of interhelical distances in the absence (blue) and in the presence (green) of an electric field. 

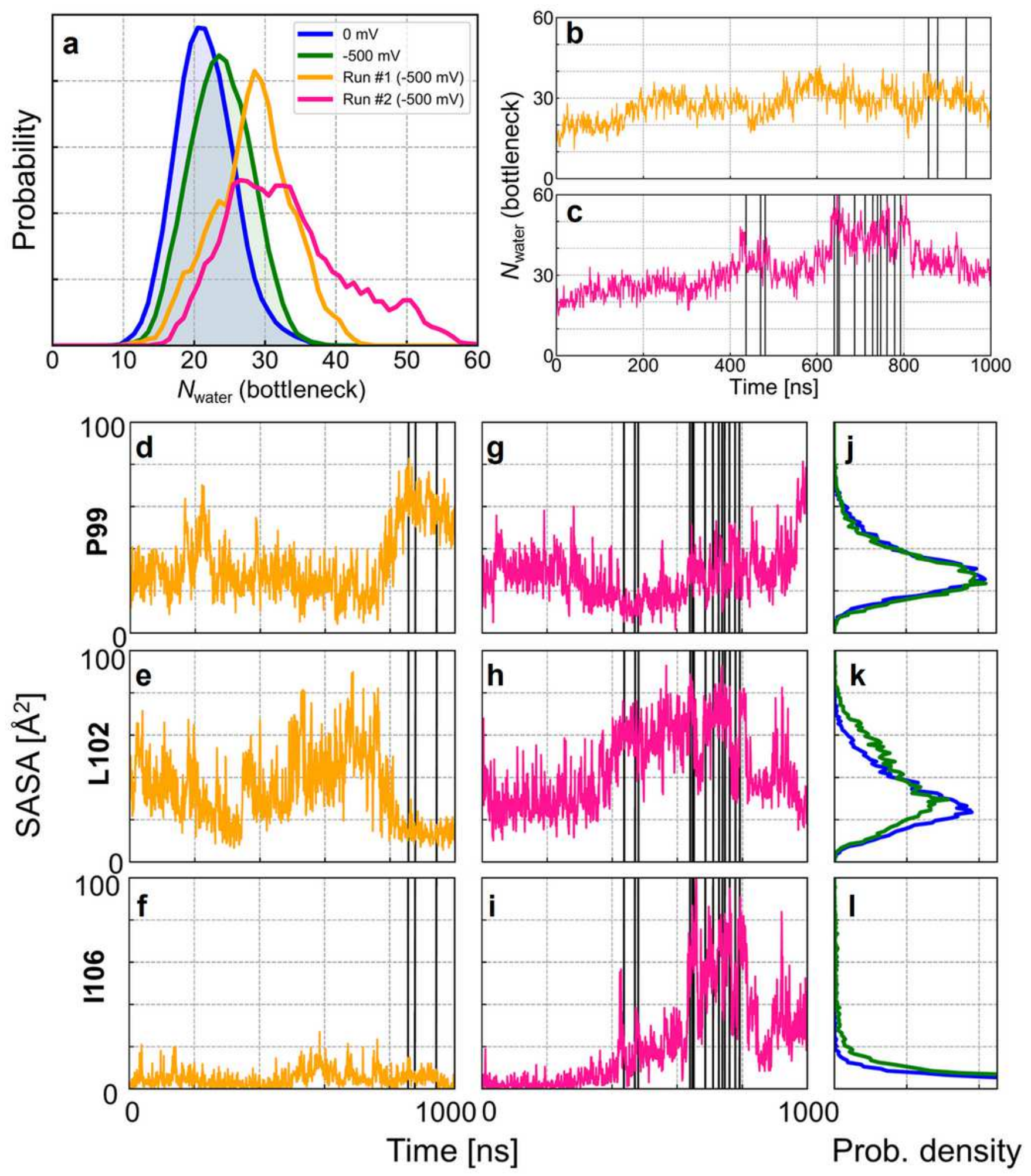

Figure 10

Analysis of hydration of the extracellular bottleneck region. (A) Distributions of the number of water molecules in the bottleneck region, Nwater(bottleneck), from nonconductive simulations in the presence (green) and absence (blue) of TM voltage. Results computed from conductive simulation runs \#1 and \#2 are shown separately. (B)-(C) Time evolution of Nwater(bottleneck) for simulation runs \#1 (orange) and 2 (pink). (D)-(I) Time series of solvent accessible surface areas (SASA) of residues P99, L102, and I106 
found in the narrowest region of the bottleneck for simulation runs \#1 [(D)-(F)] and 2 [(G)-(I)]. The time series suggest temporal correlation with ion permeation. $(J)-(L)$ Distributions of SASA of these residues in the absence (blue) and in the presence (green) of TM voltage are shown on the rightmost column. Ion translocation events (see Fig. 4) are indicated as vertical lines.
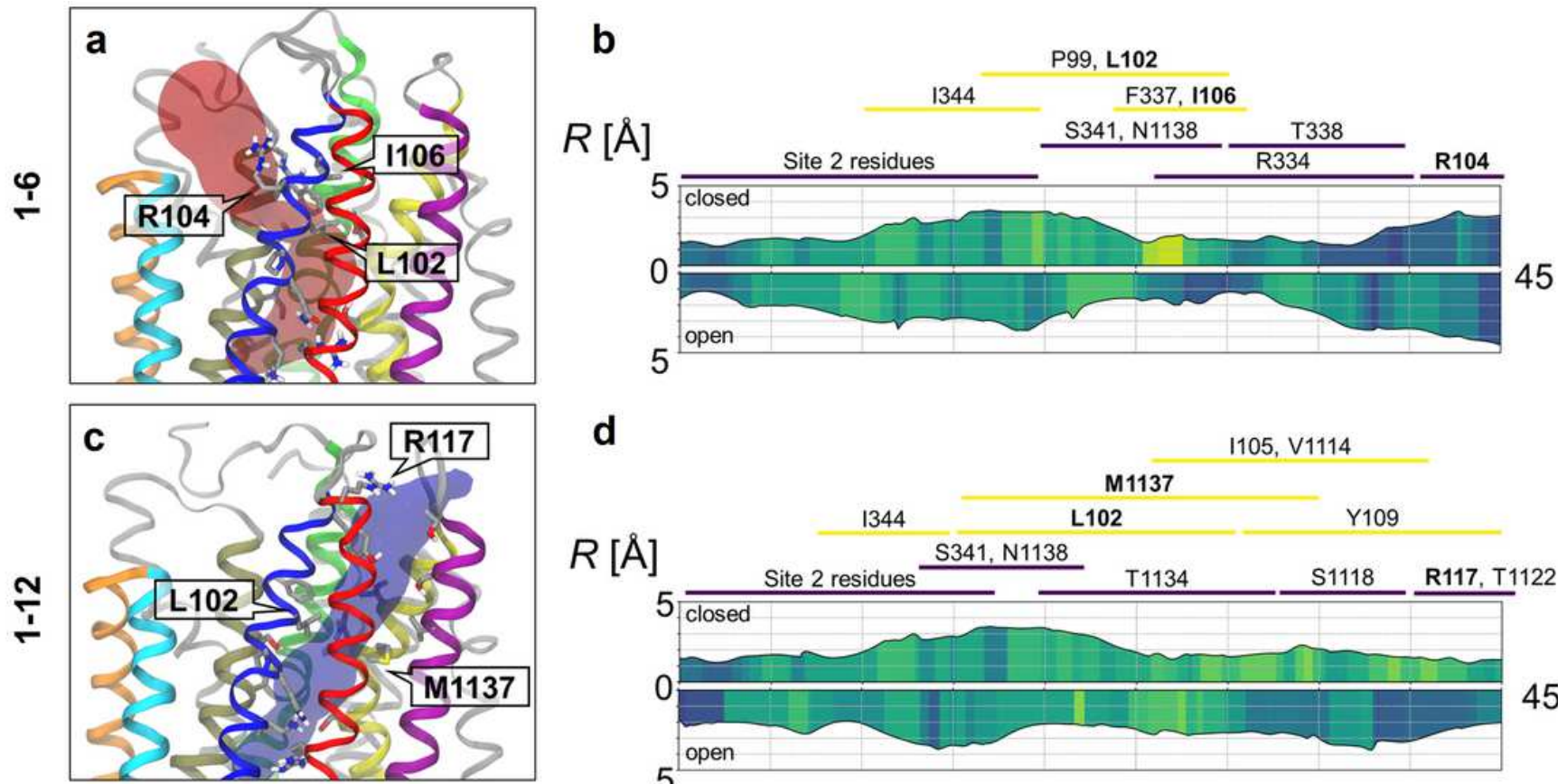

d
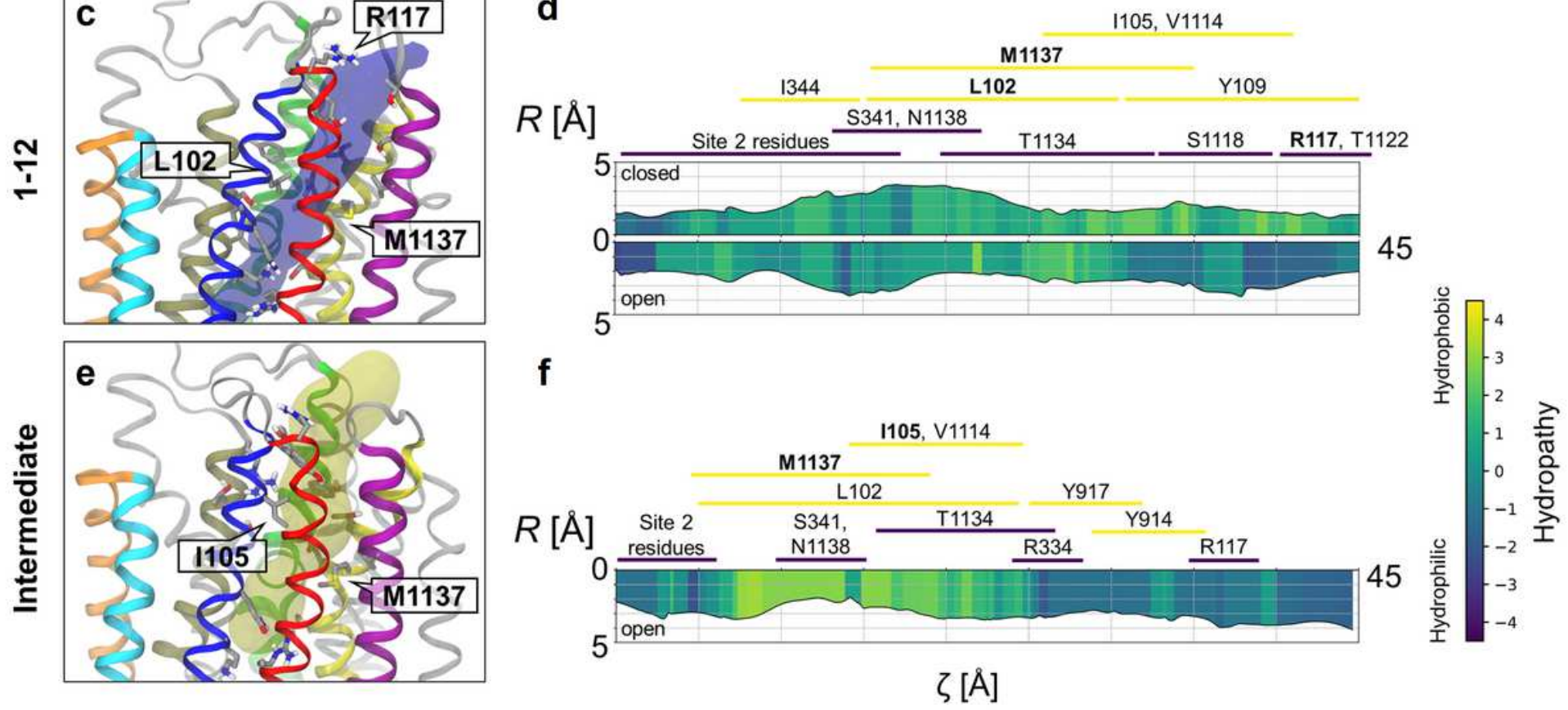

Figure 11

Structural and physico-chemical analysis of diverging permeation pathways through the extracellular bottleneck region. (A) 1-6, (C) 1-12, and (E) intermediate pathways are visualized as translucent closed surfaces. Estimates of width and hydrophobicity along the 1-6 (B), 1-12 (D), and intermediate pathways $(F)$; $R$, local radius and $\zeta$, curvilinear distance along the pathway. The comparison of closed and open states shows reduced hydrophobicity and/or increased width of the permeation pathways. Note that the intermediate pathway in the "closed" state could not be detected and analyzed. The approximate ranges of pathway-lining residues are indicated for each of the three pathways.

\section{Supplementary Files}

This is a list of supplementary files associated with this preprint. Click to download. 
- ESM.pdf 